\title{
Research on Two-Stage Hesitate Fuzzy Information Fusion Framework Incorporating Prospect Theory and Dichotomy Algorithm
}

\author{
Xiwen Tao ${ }^{1} \cdot$ Wenqi Jiang ${ }^{1}$
}

Received: 7 May 2021/Revised: 25 August 2021/Accepted: 13 October 2021/Published online: 15 January 2022

(C) Taiwan Fuzzy Systems Association 2022

\begin{abstract}
In order to control the systematic divergence among decision makers (DMs) and preserve the original decision preference, this paper proposes a novel decision information fusion framework under the hesitant fuzzy environment. First, a maximum compactness-based normalization method is presented to normalize hesitant fuzzy elements (HFEs) as pretreatment of decision data. Second, prospect theory is introduced to assign the optimal aggregation weights to maximize the efficiency of the preference aggregation process, in which the expected consensus threshold is viewed as a reference point estimated through statistic inference to distinguish DMs' status. Third, an effective feedback mechanism is designed to improve group consensus, and the dichotomy algorithm is utilized to search optimal feedback weight to preserve original decision information. Finally, a case study and comparison analysis are illustrated to show the efficiency of the proposed hesitant fuzzy information fusion method.
\end{abstract}

Keywords Group consensus - Hesitant fuzzy element . Prospect theory $\cdot$ Dichotomy algorithm

\section{Introduction}

Due to the expression and cognitive limitations of subjective decision making, fuzzy values such as interval fuzzy value [1], intuitionistic fuzzy value [2], Pythagorean fuzzy value [3] and et al., are usually used to express the DMs'

Xiwen Tao

txwfight@163.com

1 School of Economics and Management, Nanjing University of Science and Technology, Nanjing 210094, Jiangsu, China subjective attitude. In recent years, hesitant fuzzy element (HFE) especially its application in MAGDM (seen in [4-6]) have become hot topics. The diversity of group intelligence provides an essential reference for more reliable and reasonable group decision results. Meanwhile, individual diversity can also lead to systematic conflict within a group because of the difference in knowledge structure, cultural background, and risk attitude. Therefore, integrating diverse individual preferences with the required consensus level and preserving the diversity of individual preferences become an imperative topic. The literature mainly focuses on two aspects for efficient information fusion, including preference aggregation and consensus improvement.

In the individual preference aggregation process, aggregation weights assignment is the key issue. Some researchers preset aggregation weight vector subjectively (seen in [7, 8]), as a static parameter through the whole decision-making process. Considering DMs' behavior and consensus requirement, several researchers propose objective weight determination methods. (1) Behavior patternbased weight assignment, behavioral features are the basis of DMs' importance. Liu and $\mathrm{Xu}$ et al. [9] aggregated individual preference relation using a self-confidence induced ordered the weighted average operator to give more importance for those self-confident experts. Xu and Cabrerizo et al. [10] used a consistency induced ordered weighted average to collect individual preference and calculate weight vector with quantifier function (seen in [11]). (2) Optimization-based weight vector, objective weight is driven by consensus requirement. Zhang and Liang et al. [12] proposed a programming-based method to minimize maximum distance between individual and group to obtain optimal weight vector. Liu and He et al. [13] developed a maximum consensus model which maximizes 
consensus degree by adjusting the experts' weights. Xiao and Wang et al. [14] devised a maximum consensus-based optimization model combining in-degree centrality, consistency, and similarity indexes. Zhang and Pedrycz [15] established a programming model to determine DMs' weights by minimizing the distance between individual and group. There are also some other novel methods to determine weight vectors. Mohammadi and Rezaei [16] proposed a half-quadratic-based method to determine optimal weights for each individual ranking. Mohammadi and Rezaei [17] introduced a Bayesian-based method to find aggregated final weights of a group of DMs. Wan and Zhong et al. [18] constructed reference matrices of DMs to measure their weight vector by relatively closeness degree Based on the TOPSIS method. Wu and Liu et al. [19] proposed an entropy-based method to determine DMs' weights. The purpose of the individual preference fusion process is to generate reliable a group opinion that can be acceptable to DMs as far as possible.

On the aspect of group consensus improvement, linear sum method, programming-based method, and weight punishment method are applied here. (1) The first method updates individual preference by a linear sum of individual and group preference. Feedback weight is the key parameter to affect the improvement process. Considering the diversity of DMs' cooperation willingness, Cao and $\mathrm{Wu}$ et al. [20] introduced personal feedback weight to maximize harmony degree. Based on the matching of evaluation and decision environment, Perez and Cabrerizo et al. [21] proposed a dynamic feedback mechanism. With simulation-based consensus threshold, Tang and Zhou et al. [22] introduced a local linear sum method to adjust individual preference. (2) Programming-based method develops an optimization model to determine an optimal updated preference, which can often reach consensus within one interaction. Arieh and Easton [23] pointed that the adjustment of original opinion comes with a cost and proposed minimum adjustment cost model. If unit adjustment cost is constant, the minimum cost model can be transferred into the minimum adjustment distance model. Zhang and Dong et al. [24] investigated the efficiency difference of consensus improvement from the perspectives of minimum adjustment distance, elements, and DMs. Wu and Huang et al. [25] proposed a multi-stage optimization-based feedback mechanism. Xiao and Wang et al. [26] presents a two-stage consensus reaching model with minimum preference information loss. Zhang and Dong et al. [27] analyzed the equilibrium strategy to obtain optimal consensus cost of the minimum adjustment model. (3) Weight punishment method adjusts individual aggregation weights, which refuses to modify preference and to strengthen acceptability of group preference. Liu and $\mathrm{Xu}$ et al. [28] presented a dynamic weight adjustment strategy to improve group consensus. Du and Yu et al. [29] combined weight punishment and linear sum method to construct a mixed consensus improvement method. Guo and Xu et al. [30] developed a noncooperative behaviors management method based on weights modification.

The above literature improves efficiency on group information fusion. Original information preservation is a vital factor in the process. In decision practice, the consensus threshold, aggregation weights, and preference adjustment can influence the original preference preservation. However, the systematic influence is not comprehensively considered by the existing research:

(1) The consensus threshold is a crucial parameter in group decision making, which controls the start and termination of consensus improvement. Most research regards the threshold as an exogenous parameter preset before decision making, which cannot reflect the flexible consensus requirement. The exogenous threshold can lead to an overconsensus situation and force DMs to adjust opinion considerably. And it is meaningless to force DMs to adjust opinion passively to reach an excellent consensus target if a group has low-level compactness. Therefore, it is necessary to connect the consensus threshold to the preference distribution to set a reachable target for the consensus improvement process.

(2) Within the aggregation weights optimization, the importance of excess and shortage parts to the threshold is different. Generally, the shortage part will affect the adjustment volume directly in further consensus improvement. Therefore, it is essential to highlight the shortage part optimization when calculating the aggregation weights. Moreover, the differences among attributes are often ignored, which can harm the performance of the group opinion and affect the adjustment volume.

(3) Minimum adjustment distance strategy is commonly applied in the current consensus improvement. However, the existing methods may distort the preference features (individual preference direction and the order relationship of proximity), reducing the acceptance degree of adjustment advice. For example, if the original individual preference direction is positive, it is hard to force DM to accept the negative updated preference; if a DM is farthest from the group, it is impossible to force him/her to have the highest proximity degree after adjustment. Oriented to original preference preservation, the consensus improvement strategy should minimize the adjustment volume and avoid the distortion of preference features. 
In order to tackle the above problems, a two-stage hesitant fuzzy information fusion framework is developed for a high-level group consensus. The main innovative points of the research are as follows: (1) The endogenous consensus threshold: The statistic inference method estimates the expected consensus threshold rather than preset subjectively; (2) Prospect-based aggregation weight assignment: The prospect theory is introduced to calculate the optimal weight matrix to maximize the closeness on each attribute with less potential adjustment volume; (3) The dichotomy-based consensus improvement method: With the proposed method, the individual acceptance limitation is identified to prevent preference direction distortion. Moreover, the optimal feedback weights are searched through a dichotomy algorithm to minimize the adjustment distance and preserve the proximity order relationship.

The remaining parts of this paper are organized as follows: Sect. 2 introduces the concept of hesitant fuzzy MAGDM and prospect theory. Section 3 focuses on the preference aggregation process. To standardize hesitant fuzzy decision information, maximize compactness-based normalization is proposed. And then, proximity-induced hesitant fuzzy-ordered weighted averaging (P-IHFOWA) operator and its quantifier function are designed to aggregate individual preference and estimate group consensus threshold. In Sect. 4, the acceptance limit of each DM is identified firstly. Moreover, a dichotomy-based linear sum algorithm is given to improve consensus with minimum adjustment distance. Section 5 gives a numerical example and comparison analysis to verify the proposed approach and highlight its efficiency and rationality. Finally, in Sect. 6, we draw some conclusions and discuss future research possibilities.

\section{Preliminaries}

In this section, the concepts of hesitant fuzzy MAGDM and prospect theory are reviewed for further discussion.

\subsection{Hesitant Fuzzy MAGDM}

Let $A=\left\{a_{i} \mid i=1,2, \ldots, m\right\}, C=\left\{c_{j} \mid j=1,2, \ldots, n\right\}$ and $D=\left\{d_{i} \mid q=1,2, \ldots, t\right\}$ be the set of alternatives, attributes and DMs, respectively. $\quad h_{i j}^{q}=\left\{\gamma_{i j}^{q l} \mid l=1,2, \ldots, \# h_{i j}^{q}\right\}$ denotes the evaluation from $\mathrm{DM} e_{q}(q \in\{1,2, \ldots, t\}=T)$ on alternative $a_{i}(i \in\{1,2, \ldots, m\}=M)$ over attribute $c_{j}(j \in\{1,2, \ldots, n\}=N) . H^{q}=\left(h_{i j}^{q}\right)_{m \times n}$ is the evaluation matrix of DM $e_{q}(q \in T)$.
Definition $1[31,32]$. Let $X$ be a fixed set, a HFS on $X$ is in terms of a function $h_{E}$ that returns a subset of $[0,1]$, which can be defined as follows: $E=\left\{\left\langle x, h_{E}(x)\right\rangle\right.$ $\mid x \in X\} . h_{E}(x)$ denotes the possible membership degrees of the element $x \in X$ to the set $E$, which is a set of values in $[0,1][0,1]$. For convenience, $h_{E}(x)$ is called a $H F E$ denoted as follows: $h_{E}(x)=\left\{\gamma^{l} \mid l=1,2, \ldots, \# h_{E}(x)\right\}$, where $\# h_{E}(x)$ denotes the number of elements. Generally, elements in $h_{E}(x)$ are sorting in ascending order.

Remark 1 Considering the correspondence of linguistic term $s_{t}(t=-\tau, \ldots,-1,0,1, \ldots, \tau) \quad$ with membership $\gamma(\gamma \in[0,1])[33],-\tau \leq s^{l}<0$ and $0 \leq \gamma<0.5$ share the same negative attitude. On the contrary, $0<s^{l} \leq \tau$ and $0.5<\gamma^{l} \leq 1$ show the opposite attitude [34]. For example, considering quality of service as a linguistic variable, $0<s^{l} \leq \tau$ means that the service quality is good. Accordingly, $0.5<\gamma^{l} \leq 1$ also represents DM's positive attitude. In real situations, it is difficult for DMs to transform original attitude absolutely.

Definition 2 [32] Let $h=\left\{\gamma^{l} \mid l=1,2, \ldots, \# h\right\}$ be a HFE, $\gamma^{1}$ and $\gamma^{\# h}$ represent the minimum and maximum values in $h$, respectively. Then, $\tilde{\gamma}=\xi \gamma^{1}+(1-\xi) \gamma^{\# h}$ is the artificial value of $h$, where parameter $\xi \in[0,1]$.

For the difference of DMs' hesitancy, a number of elements in HFE are not the same. To compare and aggregate HFEs, normalization process is usually necessary. In this paper, artificial elements $\tilde{\gamma}=\xi \gamma^{1}+(1-\xi) \gamma^{\# h}$ are added to normalize HFE. $\bar{H}^{q}=\left(\bar{h}_{i j}^{q}\right)_{m \times n}$ denotes the normalized matrix, where $\bar{h}_{i j}^{q}=\left\{\bar{\gamma}_{i j}^{q l} \mid l=1,2, \ldots, L\right\}$ and $L=\max \left\{\# h_{i j}^{q} \mid i \in M, j \in N, q \in T\right\}$.

Remark 2 According to Ref.[35], two opposite principles are employed for the HFE normalization: (1) The $\alpha$-normalization: The information loss is inevitable because some elements will be deleted until all HFEs have the same length. Furthermore, the information loss in the process is irreversible in the further information fusion stage. (2) The $\beta$-normalization: The adding artificial element is the convex combination of original preferences. Compared with the $\alpha$-normalization, it can avoid original information loss as far as possible because all elements are preserved. However, the DMs' subjective randomness in determining the artificial elements may result in information distortion. Therefore, in the following, a maximizing group compactness rule is proposed to guide the $\beta$-normalization process to reduce subjective randomness. 
Definition 3 [36] Let $\bar{h}_{1}$ and $\bar{h}_{2}$ be two HFEs, similarity between $\bar{h}_{1}$ and $\bar{h}_{2}$ is calculated based on distance measurement:

$S\left(\bar{h}_{1}, \bar{h}_{2}\right)=1-\sqrt{\sum_{l=1}^{L}\left(\bar{\gamma}_{1}^{l}-\bar{\gamma}_{2}^{l}\right)^{2}} / L$.

In MAGDM problem, similarity between $\bar{H}^{q}=\left(\bar{h}_{i j}^{q}\right)_{m \times n}$ and $\bar{H}^{u}=\left(\bar{h}_{i j}^{u}\right)_{m \times n}$ is calculated as follows:

$S\left(\bar{H}^{q}, \bar{H}^{u}\right)=1-\sum_{j=1}^{n} \sum_{i=1}^{m} \sqrt{\sum_{l=1}^{L}\left(\bar{\gamma}_{i j}^{q l}-\bar{\gamma}_{i j}^{u l}\right)^{2}} / L m n$

To collect HFEs, several aggregation operators are designed. Among them, induced hesitant fuzzy-ordered weight average (IFHOWA) operator can control aggregation process more targeted [10]:

Definition 4 [10] Let IFHOWA be the mapping $\Omega^{n} \rightarrow \Omega$, where $\boldsymbol{w}=\left(w_{1}, w_{2}, \ldots, w_{n}\right)^{T}\left(\sum_{j=1}^{n} w_{j}=1, w_{j} \geq 0\right)$ is the associated weight vector of IFHOWA. $\sigma$ is a permutation of $\{1,2, \ldots, n\}$ such that $u_{\sigma(j)} \geq u_{\sigma(j+1)}, \forall j=1,2, \ldots, n-1$, i.e., $\left\langle u_{\sigma(j)}, \bar{h}_{\sigma(j)}\right\rangle$ is the 2-tuple with $u_{\sigma(j)}$ the $j$ th largest value in $\left\{u_{1}, u_{2}, \ldots, u_{n}\right\}$. IFHOWA operator are defined as follows:

$$
\begin{aligned}
& \text { IHFOWA } \\
& \left.\left.=\bar{\gamma}_{\sigma(1)} \in \bar{h}_{\sigma(1)}, \bar{\gamma}_{\sigma(2)} \in \bar{h}_{\sigma(2)}, \ldots, \bar{\gamma}_{\sigma(n)} \in \bar{h}_{\sigma(n)}\right\rangle,\left\langle u_{2}, \bar{h}_{2}\right\rangle, \ldots,\left\langle u_{n}, \bar{h}_{n}\right\rangle\right) \\
& \left.\cup\left(w_{j} \bar{\gamma}_{\sigma(j)}\right)\right\} .
\end{aligned}
$$

IHFOWA operator takes $\left\langle u_{\sigma(j)}, \bar{h}_{\sigma(j)}\right\rangle$ as its argument pairs, in which the second component $\bar{h}_{\sigma(j)}$ is reordered according to the first component $u_{\sigma(j)}$ referred to induced variable.

\subsection{Prospect Theory}

Prospect theory depicts the behavior of choosing prospects in the presence of risk. Each prospect, related to risk context, is viewed as a potential gain or loss [37]. People with different behavior patterns will choose different decision frameworks based on different prospects. Since prospect theory can illustrate psychological phenomena flexibly, it is widely used in decision analysis. $\mathrm{Wu}$ and $\mathrm{Li}$ et al. [38] used prospect theory to calculate the satisfaction of suppliers to evaluate the cooperation performance. Wang et al. [39] proposed a prospect theory-based MAGDM method with stochastic uncertainty. In the consensus reaching process, the similarity between individual and group opinion represents DMs' consensus state. Specifically, a preference that is beyond the consensus threshold is seen as a potential gain. On the contrary, if preference cannot reach consensus requirement, it will be seen as potential loss due to time consumption and information loss during opinion adjustment. Practically, the purpose of information fusion is to obtain potential gain and avoid loss as far as possible. The gap between individual and consensus threshold influences consensus cost more significantly compared with the excess part. The consensus threshold is thus regarded as a reference point providing a context to classify DMs' potential consensus state.

The value function is the key part of prospect theory, which represents the gain or loss depending on the reference point. $x>0$ means gain and $x<0$ means loss. $\alpha$ and $\beta$ are the risk attitude parameters $(0 \leq \alpha, \beta \leq 1)$. Larger risk attitude parameters mean that DM tends to be risk-seeking. $\lambda$ is the risk aversion parameter, where $\lambda>1$ means that DM is more sensitive to loss. The prospect value function is

$v(x)=\left\{\begin{array}{l}x^{\alpha}, x>0 \\ -\lambda(-x)^{\beta}, x \leq 0\end{array}\right.$

Loss area has higher slope compared with gain area in the value function, representing that people are often more susceptive to losses, i.e., the pain of losses is much greater than the pleasure of gains.

\section{Preference Aggregation Based on Prospect Theory}

In this section, a novel preference aggregation method is proposed based on prospect theory. As the pretreatment of decision preference, a novel HFEs normalization method is developed to optimize group compactness. And then, statistic inference is employed to connect the preference distribution to the expected consensus threshold. Finally, the optimal weights are determined by maximizing comprehensive prospects for a high-level group consensus.

\subsection{Compactness-Based HFE Normalization}

In order to compare and aggregate HFEs, a normalization process is necessary. There are few clear rules guiding the normalization process in the field of hesitant fuzzy MAGDM. In the following, HFEs will be normalized by maximizing compactness, which is measured by the proximity index.

According to Definition 3, proximity index $P I_{i j}^{q}$ is

$P I_{i j}^{q}=\sum_{u \neq q} S\left(\bar{h}_{i j}^{q}, \bar{h}_{i j}^{u}\right) / t-1$,

which represents the average compactness between $e^{q}$ and the others on $a_{i}$ over $c_{j}$. The proximity index $P I_{i j}$ is 
$P I_{i j}=\sum_{q=1}^{t} \sum_{u \neq q}^{t} S\left(\bar{h}_{i j}^{q}, \bar{h}_{i j}^{u}\right) / t(t-1)$

which represents the average closeness degree on $a_{i}$ over $c_{j}$. It is easy to prove that $0 \leq P I_{i j}^{q}, P I_{i j} \leq 1$. In this paper, local normalization parameter $\xi_{i j}^{q}(i \in M, j \in N, q \in T)$ is utilized to maximize group compactness with following programming model:

$$
(M-1)\left\{\begin{array}{l}
\operatorname{Max} \sum_{j=1}^{n} \sum_{i=1}^{m} P I_{i j} / m n \\
\text { s.t. }\left\{\begin{array}{l}
\bar{h}_{i j}^{q}=\left\{\bar{\gamma}_{i j}^{q 1}, \xi_{i j}^{q} \bar{\gamma}_{i j}^{q 1}+\left(1-\xi_{i j}^{q}\right)_{i j}^{q \# \bar{\gamma}_{i j}^{q}}, \ldots, \bar{\gamma}_{i j}^{q \# \bar{h}_{i j}^{q}}\right\}, \\
0 \leq \xi_{i j}^{q} \leq 1, i \in M, j \in N, q \in T
\end{array}\right.
\end{array}\right.
$$

where $\xi_{i j}^{q *}$ denotes optimal normalization parameter from $(M-1)$. Compared with traditional risk attitude-based normalization [36], the proposed method can enhance the closeness among individuals, which is beneficial for further aggregation process.

\subsection{Expected Consensus Threshold Estimation}

In this sub-section, proximity-induced hesitant fuzzyordered weighted averaging (P-IHFOWA) operator and its associated quantifier function is designed to calculate individual consensus range on each position. And then, T-distribution is applied to estimate the expected consensus threshold.

Definition 5 Let P-IFHOWA be the mapping $\Omega^{t} \rightarrow \Omega$, where $\boldsymbol{w}_{i j}=\left(w_{i j}^{1}, w_{i j}^{2}, \ldots, w_{i j}^{t}\right)^{T} \quad\left(\sum_{q=1}^{t} w_{i j}^{q}=1, w_{i j}^{q} \geq 0\right) \quad$ is their corresponding weight vector of P-IFHOWA. $\sigma$ is a permutation of $\{1,2, \ldots, n\}$ such that $P I_{i j}^{\sigma(q)} \geq P I_{i j}^{\sigma(q+1)}$, $\forall q=1,2, \ldots, t-1$, i.e.. $\left\langle P I_{i j}^{\sigma(q)}, \bar{h}_{i j}^{\sigma(q)}\right\rangle$ is the 2-tuple with $P I_{i j}^{\sigma(q)}$ the $q$ th largest value in $\left\{P I_{i j}^{\sigma(1)}, P I_{i j}^{\sigma(2)}, \ldots, P I_{i j}^{\sigma(t)}\right\} . \mathrm{P}-$ IFHOWA operator is defined as follows:

$$
\begin{aligned}
P & -\operatorname{IHFOWA} A_{w}\left(\left\langle P I_{i j}^{1}, \bar{h}_{i j}^{1}\right\rangle,\left\langle P I_{i j}^{2}, \bar{h}_{i j}^{2}\right\rangle, \ldots,\left\langle P I_{i j}^{t}, \bar{h}_{i j}^{t}\right\rangle\right) \\
& =\sum_{j=1}^{n} h_{i j}^{\sigma(q)} w_{i j}^{q} \\
& ={ }_{\bar{\gamma}_{i j}^{\sigma(1) l} \in \bar{h}_{i j}^{\sigma(1)}, \bar{\gamma}_{i j}^{((2))} \in \bar{h}_{i j}^{\sigma(2) l}, \ldots, \bar{\gamma}_{i j}^{\sigma(t) l} \in \bar{h}_{i j}^{\sigma(t) l}}\left\{\left(w_{i j}^{q} \bar{\gamma}_{i j}^{\sigma(q) l}\right)\right\} .
\end{aligned}
$$

The weight vector is calculated by quantifier function:

$$
w_{i j}^{\sigma(q)}=\left(\sum_{k=1}^{q} P I_{i j}^{\sigma(k)} / t P I_{i j}\right)^{a_{i j}}-\left(\sum_{k=1}^{q-1} P I_{i j}^{\sigma(k)} / t P I_{i j}\right)^{a_{i j}} \text {, }
$$

where $a_{i j} \in[0,1]$ denotes the quantifier parameter.

Example 1 For an alternative under a criterion, four DMs are invited to provide evaluation values: $h^{1}=\{0.2,0.4\}, h^{2}=\{0.7,0.7\}, \quad h^{3}=\{0.4,0.5\}$, $h^{4}=\{0.5,0.8\}$. Based on Eq. (5), $P I^{q}(q=1,2,3,4)$ are
$P I^{1}=0.782, \quad P I^{2}=0.805, P I^{3}=0.850, P I^{4}=0.826$, $P I=0.816$. Rank the proximity indexes in decreasing order and we get $\left\{P I^{3}, P I^{4}, P I^{2}, P I^{1}\right\}$. According to the proximity index order, the aggregation weights are calculated by the following formula:

$$
\begin{aligned}
w^{3}= & \left(P I^{3} / 4 P I\right)^{a}, w^{4}=\left(\left(P I^{3}+P I^{4}\right) / 4 P I\right)^{a}-\left(P I^{3} /\right. \\
& 4 P I)^{a}, \quad w^{2}=\left(\left(P I^{3}+P I^{4}+P I^{2}\right) / 4 P I\right)^{a}- \\
& \left(\left(P I^{3}+P I^{4}\right) / 4 P I\right)^{a}, \\
w^{1}= & \left(\left(P I^{3}+P I^{4}+P I^{2}+P I^{1}\right) / 4 P I\right)^{a}-\left(\left(P I^{3}+P I^{4}+P I^{2}\right) / 4 P I\right)^{a} .
\end{aligned}
$$

Namely, $\quad w^{3}=(0.260)^{a}, \quad w^{4}=(0.513)^{a}-(0.260)^{a}$, $w^{2}=(0.760)^{a}-(0.513)^{a}$, and $w^{1}=1-(0.760)^{a}$.

In this paper, quantifier parameter $a$ is optimized to maximize the individual consensus level (seen the following model $(M-3))$.

Remark 3 With P-IHFOWA operator, larger weight will be assigned to DMs who is closer to the others, i.e., $w_{i j}^{\sigma(q)} \geq w_{i j}^{\sigma(q+1)}, \forall q=1,2, \ldots, t-1$. It is difficult for moderator to determine all DMs' weight subjectively. For convenience of calculation, quantifier function is used (see Eq. (8)) to reduce the number of variables. With given proximity index, quantifier parameter $a_{i j}$ is the only factor affecting weight. individual preferences can then be aggregated into group opinion $\bar{H}^{c}=\left(\bar{h}_{i j}^{c}\right)_{m \times n}$, where

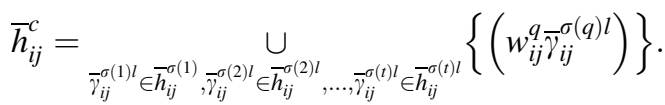

Individual consensus index $C I_{i j}^{q}$ is calculated as follows:

$C I_{i j}^{q}=S\left(\bar{h}_{i j}^{c}, \bar{h}_{i j}^{q}\right)=1-\sqrt{\sum_{l=1}^{L}\left(\bar{\gamma}_{i j}^{q l}-\bar{\gamma}_{i j}^{c l}\right)^{2}} / L$,

where $C I_{i j}^{q}$ denotes similarity between DM $e_{q}$ with the group on $a_{i}$ over $c_{j}$, which varies from quantifier parameter $\alpha_{i j}$. And then, individual consensus range $\left[C I_{i j}^{q-}\right.$, $\left.C l_{i j}^{q+}\right]=\left[\operatorname{Min} I_{i j}^{q}, \operatorname{Max} \mathrm{CI}_{i j}^{q}\right]$ is extracted by the following programming model:

$$
(M-2)\left\{\begin{array}{c}
\operatorname{Max} / \operatorname{Min} I_{i j}^{q}=1-\sqrt{\sum_{l=1}^{L}\left(\bar{\gamma}_{i j}^{q}-\bar{\gamma}_{i j}^{c}\right)^{2}} / L \\
\text { s.t. }\left\{\begin{array}{c}
\bar{\gamma}_{i j}^{c}=w_{i j}^{q} \bar{\gamma}_{i j}^{\sigma(q) l} \\
w_{i j}^{\sigma(q)}=\left(\sum_{k=1}^{q} P I_{i j}^{\sigma(k)} / t P I_{i j}\right)^{a_{i j}} \rightarrow \\
\quad-\left(\sum_{k=1}^{q-1} P I_{i j}^{\sigma(k)} / t P I_{i j}\right)^{a_{i j}} \\
0 \leq a_{i j} \leq 1, i \in M, j \in N, q \in T
\end{array}\right.
\end{array}\right.
$$

For $\bar{H}^{q}=\left(\bar{h}_{i j}^{q}\right)_{m \times n}, C I_{i j}^{q}$ is a bounded and continuous function of $a_{i j}$, i.e., $C I_{i j}^{q}=f\left(a_{i j}\right)$. Due to $a_{i j} \in[0,1]$, there exists feasible solution for $(M-2)$. Minimum/Maximum individual consensus index sequence $\left\{C I_{i j}^{1-}, C I_{i j}^{1+}, C I_{i j}^{2-}, C I_{i j}^{2+}, \ldots, C I_{i j}^{t-}, C I_{i j}^{t+}\right\}$ is viewed as a set 
of sample data. Suppose individual consensus index sequence obeys normal distribution approximately, the sample average $\overline{C I}_{i j}$ and sample variance $s_{i j}^{2}$ are

$\overline{C I}_{i j}=\frac{1}{2 t}\left(\sum_{q=1}^{t} C I_{i j}^{q-}+\sum_{q=1}^{t} C I_{i j}^{q+}\right)$,

$s_{i j}^{2}=\frac{1}{2 t-1}\left(\sum_{q=1}^{t}\left(C I_{i j}^{q-}-\overline{C I}_{i j}\right)^{2}+\sum_{q=1}^{t}\left(C I_{i j}^{q+}-\overline{C I}_{i j}\right)^{2}\right)$.

Since total variance is unknown, T-distribution is applied to estimate probability of group consensus range $\left[C I_{i j}^{q-}, C I_{i j}^{q+}\right]$ :

$P_{i j}^{q}=P\left(t \leq C I_{i j}^{q+}\right)-P\left(t \leq C I_{i j}^{q-}\right)$,

where $P\left(t \leq C I_{i j}^{q+}\right)=1-P\left(\frac{C l_{s_{j}^{+}}^{q+}-\bar{C} I_{i j}}{s_{i j} / \sqrt{2 t}}\right) ; P\left(t \leq C I_{i j}^{q-}\right)=1-P\left(\frac{C l_{i j}^{q-}-\overline{I_{i j}}}{s_{i j} / \sqrt{2 t}}\right)$.

Expected consensus index range on $a_{i}$ over $c_{j}$ is $\left[E C I_{i j}^{-}, E C I_{i j}^{+}\right]$:

$E C I_{i j}^{-}=\sum_{q=1}^{t}\left(P_{i j}^{q} / \sum_{q=1}^{t} P_{i j}^{q}\right) C I_{i j}^{q-}$,

$E C I_{i j}^{+}=\sum_{q=1}^{t}\left(P_{i j}^{q} / \sum_{q=1}^{t} P_{i j}^{q}\right) C I_{i j}^{q+}$.

Therefore, expected consensus threshold on $a_{i}$ over $c_{j}$ is $E C I_{i j}=\eta E C I_{i j}^{-}+(1-\eta) E C I_{i j}^{+},(\eta \in[0,1])$.

\subsection{Aggregation Weight Vector Calculation}

Prospect theory is applied to depict DMs' consensus state during aggregation process, where expected consensus threshold $E C I_{i j}$ is seen as reference point. $C I_{i j}^{q} \geq E C I_{i j}$ means the DM $e_{q}$ has potential gain on alternative $a_{i}$ over attribute $c_{j}$, whereas its counterpart represents potential loss. The prospect value of $e_{q}$ on $a_{i}$ over $c_{j}$ is

$v_{i j}^{q}=\left\{\begin{array}{l}\left(C I_{i j}^{q}-E C I_{i j}\right)^{\alpha},\left(C I_{i j}^{q}-E C I_{i j}\right) \geq 0 \\ -\lambda\left(-\left(C I_{i j}^{q}-E C I_{i j}\right)\right)^{\beta},\left(C I_{i j}^{q}-E C I_{i j}\right)<0\end{array}\right.$,

where $\alpha=\beta=0.88, \lambda=2.25[37]$. The collective consensus prospect value $p v_{i j}$ is calculated by $p v_{i j}=\sum_{q=1}^{t} w_{i j}^{q} v_{i j}^{q}$, where $w_{i j}^{q}$ is the decision weight calculated by Eq. (8). Naturally, an optimal weight vector $w_{i j}=\left(w_{i j}^{1}, w_{i j}^{2}, \ldots, w_{i j}^{t}\right)^{T}$ is needed to maximize comprehensive prospect value, a programming model is, therefore, developed for optimal aggregation weight vector:
$(M-3)\left\{\begin{array}{l}\operatorname{Max} P=\sum_{j=1}^{n} \sum_{i=1}^{m} p v_{i j} \\ \text { s.t. }\left\{\begin{array}{l}v_{i j}^{q}=\left\{\begin{array}{l}\left(C I_{i j}^{q}-E C I_{i j}\right)^{\alpha},\left(C I_{i j}^{q}-E C I_{i j}\right)>0 \\ -\lambda\left(-\left(C I_{i j}^{q}-E C I_{i j}\right)\right)^{\beta},\left(C I_{i j}^{q}-E C I_{i j}\right) \leq 0\end{array}\right. \\ C I_{i j}^{q}=1-\sqrt{\sum_{l=1}^{L}\left(\bar{\gamma}_{i j}^{q}-\left(1-\prod_{j=1}^{n}\left(1-\bar{\gamma}_{i j}^{\sigma(q) l}\right)^{w_{i j}^{q}}\right)\right)^{2}} / L \\ w_{i j}^{\sigma(q)}=\left(\sum_{k=1}^{q} P I_{i j}^{\sigma(k)} / t P I_{i j}\right)^{a_{i j}}-\left(\sum_{k=1}^{q-1} P I_{i j}^{\sigma(k)} / t P I_{i j}\right)^{a_{i j}} \\ 0 \leq a_{i j} \leq 1, i \in M, j \in N, q \in T\end{array}\right.\end{array}\right.$

where $a_{i j}^{*}$ and $w_{i j}^{q *}=\left(w_{i j}^{1 *}, w_{i j}^{2 *}, \ldots, w_{i j}^{t *}\right)^{T}$ denote optimal quantifier parameter and weight vector respectively. Plugging $w_{i j}^{q *}$ into Eq. (9), optimal group preference $\bar{H}^{c *}=$ $\left(\bar{h}_{i j}^{c *}\right)_{m \times n}$ with maximum comprehensive prospect is generated.

Example 2 Apply the Example 1 to illustrate the process of weight vector optimization. First, model $(M-2)$ is applied to determine the estimated consensus threshold $E C I$, where

$$
\begin{aligned}
& \gamma \prime^{c}=1-(1-0.2)^{w^{1}}(1-0.7)^{w^{2}}(1-0.4)^{w^{3}}(1-0.5)^{w^{4}}, \\
& \gamma / \prime^{c}=1-(1-0.4)^{w^{1}}(1-0.7)^{w^{2}}(1-0.5)^{w^{3}}(1-0.8)^{w^{4}} \\
& M-2.1)\left\{\begin{array}{l}
\operatorname{Min} / \operatorname{MaxCI}^{1}=1-\sqrt{\left(0.2-\gamma /{ }^{c}\right)^{2}+\left(0.4-\gamma / \prime^{c}\right)^{2}} / 2 \\
w^{1}=1-(0.760)^{a}, 0 \leq a \leq 1
\end{array}\right.
\end{aligned}
$$

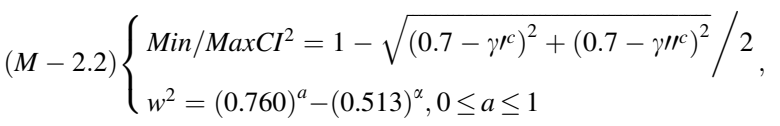

$$
\begin{aligned}
& (M-2.3)\left\{\begin{array}{l}
\operatorname{Min} / \operatorname{Max} C^{3}=1-\sqrt{\left(0.4-\gamma /{ }^{c}\right)^{2}+\left(0.5-\gamma / \prime^{c}\right)^{2}} / 2 \\
w^{3}=(0.260)^{a}, 0 \leq a \leq 1
\end{array}\right. \\
& (M-2.4)\left\{\begin{array}{l}
\operatorname{Min} / \operatorname{MaxCI}^{4}=1-\sqrt{\left(0.5-\gamma / c^{c}\right)^{2}+\left(0.8-\gamma / \prime^{c}\right)^{2}} / 2 \\
w^{4}=(0.513)^{a}-(0.260)^{a}, 0 \leq a \leq 1
\end{array}\right. \\
& {\left[\mathrm{CI}^{q-}, \mathrm{CI}^{q+}\right](q=1,2,3,4) \quad \text { are } \quad[0.816,0.888] \text {, }}
\end{aligned}
$$

$$
\left\{\begin{array}{l}
\text { s.t. }\left\{\begin{array}{l}
\text { MaxP }=\sum_{q=1}^{4} w^{q} v^{q} \\
C I^{3}=1-\sqrt{\left(0.2-\gamma^{c}\right)^{2}+\left(0.4-\gamma^{c}\right)^{2}} / 2, C I^{2}=1-\sqrt{\left(0.4-\gamma^{c}\right)^{2}+\left(0.5-\gamma^{c}\right)^{2}} / 2, C I^{4}=1-\sqrt{\left(0.5-\gamma^{c}\right)^{2}+\left(0.8-\gamma^{c}\right)^{2}} / 2 \\
v^{q}=\left\{\begin{array}{l}
\left(C I^{q}-0.847\right)^{\alpha}, C I^{q}>0.847 \\
-\lambda\left(0.847-C I^{q}\right)^{\beta}, C I^{q} \leq 0.847 \\
0 \leq a \leq 1, q=1,2,3,4
\end{array}\right.
\end{array},\left\{\begin{array}{l}
w^{1}=1-(0.760)^{a}, w^{2}=(0.760)^{a}-(0.513)^{a} \\
w^{3}=(0.260)^{a}, w^{4}=(0.513)^{a}-(0.260)^{a}
\end{array}\right.\right.
\end{array}\right.
$$

With $(M-3)$, we have $a=0.245$. And the optimal aggregation weights are

$w^{1}=0.0649, w^{2}=0.0857, w^{3}=0.7191, w^{4}=0.1302$. 


\section{Consensus Improvement Based on Dichotomy Algorithm}

Consensus improvement is a significant process to enhance the acceptability of group preference. In this section, an identification rule is firstly proposed to select adjusted preferences. In order to prevent distorting DMs' attitudes during the adjustment process, the feasible range of feedback weight is calculated for the improved linear sum method. And then, a dichotomy-based optimal weight searching algorithm is designed to update individual preference.

\subsection{Adjusted Preferences Identification}

With the optimal group preference $\bar{H}^{c *}=\left(\bar{h}_{i j}^{c *}\right)_{m \times n}$, group consensus index $G C I_{i j}$ on $a_{i}$ over $c_{j}$ is calculated as follows:

$G C I_{i j}=\sum_{q=1}^{t} C I_{i j}^{q *} / t$,

where $C I_{i j}^{q *}$ denotes individual consensus index with $\bar{h}_{i j}^{c *}$. The group preference $\bar{H}^{c *}=\left(\bar{h}_{i j}^{c *}\right)_{m \times n}$ is regarded as completely acceptable only if $G C I_{i j} \geq E C I_{i j}$ for all $i \in M, j \in N$. Otherwise, it is necessary to adjust individual preference.

For further adjustment, an identification rule is developed to select modified positions. Let binary array $(i, j)$ denote the position for alternative $a_{i}$ over attribute $c_{j}$. Identification rules (IRs) are described as follows.

IR: The set of positions named Pos that should enhance group consensus level $G C I_{i j}$ which is lower that the threshold $E C I_{i j}$. It is calculated by

Pos $=\left\{(i, j) \mid G C I_{i j}<E C I_{i j}\right\}$.

Let $K$ denote the number of positions to be adjusted. Once Pos has been identified, feedback process is activated to provide DMs adjustment recommendation.

\subsection{Feedback Weight Optimization}

With adjusted positions, the linear sum method is applied to update individual preferences. The traditional methods have not provided a mechanism to control the adjustment distance and prevent distorting DMs' attitudes. The purpose of the sub-section is to search for the optimal feedback weight to avoid over-adjustment and preserve DMs' original attitude. With the linear sum method, the updated preference is

$\bar{h} \prime_{i j}^{q}=\mu_{i j} \bar{h}_{i j}^{q}+\left(1-\mu_{i j}\right) \bar{h}_{i j}^{c *}$

where $\mu_{i j} \in[0,1)$ and $(i, j) \in \operatorname{Pos}, q \in T$. The larger $\mu_{i j}$ is, the less group opinion will be accepted. On the contrary, small feedback weight means DM accepts group preference to a greater extent.

Theorem 1 Let PII ${ }_{i j}^{q}$ denote the updated proximity index of DM $e_{q}$ on $a_{i}$ over $c_{j}$, then

$$
\left(P I I_{i j}^{q}-P I I_{i j}^{p}\right)\left(P I_{i j}^{q}-P I_{i j}^{p}\right)>0, q \neq p .
$$

Proof The updated proximity index is $P I I_{i j}^{q}=\sum_{u \neq q} S$ $\left(\bar{h}_{i j}^{q}, \bar{h}_{i j}^{u}\right) / t-1$. Plugging Eq. (1) into it, PI $\prime_{i j}^{q}$ can be rewritten as follows:

$P I I_{i j}^{q}=1-\sum_{u \neq q} \sqrt{\sum_{l=1}^{L}\left(\left(\mu_{i j} \gamma_{i j}^{l}+\left(1-\mu_{i j}\right) \bar{\gamma}_{i j}^{c * l}\right)-\left(\mu_{i j} \bar{\gamma}_{i j}^{l l}+\left(1-\mu_{i j}\right) \bar{\gamma}_{i j}^{c * l}\right)\right)^{2}} / L(t-1)$,

$P I \prime_{i j}^{q}=1-\mu_{i j} \sum_{u \neq q} \sqrt{\sum_{l=1}^{L}\left(\bar{\gamma}_{i j}^{q l}-\bar{\gamma}_{i j}^{u l}\right)^{2}} / L(t-1)$

Q.E.D.

We have

$$
\begin{aligned}
& P I_{i j}^{q}-P I_{i j}^{p}=-\mu_{i j}\left(\sum_{u \neq q} \sqrt{\sum_{l=1}^{L}\left(\bar{\gamma}_{i j}^{q}-\bar{\gamma}_{i j}^{u l}\right)^{2}}-\sum_{u \neq q} \sqrt{\sum_{l=1}^{L}\left(\bar{\gamma}_{i j}^{p l}-\bar{\gamma}_{i j}^{u l}\right)^{2}}\right) / L(t-1), \\
& P I_{i j}^{q}-P I_{i j}^{p}=-\left(\sum_{u \neq q} \sqrt{\sum_{l=1}^{L}\left(\bar{\gamma}_{i j}^{q l}-\bar{\gamma}_{i j}^{u l}\right)^{2}}-\sum_{u \neq q} \sqrt{\sum_{l=1}^{L}\left(\bar{\gamma}_{i j}^{p l}-\bar{\gamma}_{i j}^{u l}\right)^{2}}\right) / L(t-1) .
\end{aligned}
$$

It follows that $\left(P I I_{i j}^{q}-P I I_{i j}^{p}\right)\left(P I_{i j}^{q}-P I_{i j}^{p}\right)>0, q \neq p$.

The above analysis means that proximity feature of updated preference by Eq. (1) remains the same as the original. When updated preference is aggregated by $\mathrm{P}$ IOWA operator, the original weight vectors also satisfy that DMs with higher proximity will be assigned more weight. Therefore, for computation convenience and convergence, it is reasonable to assign the original weight vector to updated preference. The updated group preference is $\bar{H} \prime^{c}=\left(\bar{h}_{i j}^{c}\right)_{m \times n}$, where

$\bar{h}_{i j}^{c}=\sum_{q=1}^{t} w_{i j}^{q}\left(\mu_{i j} \bar{h}_{i j}^{q}+\left(1-\mu_{i j}\right) \bar{h}_{i j}^{c *}\right)$

and then, we prove the convergency of updated strategy.

Theorem 2 Let $G C I_{i j}^{\prime}$ denote the updated group proximity index on $a_{i}$ over $c_{j}$, then $G C I \prime_{i j}>G C I_{i j}$.

Proof The updated group consensus index is $G C I \prime_{i j}=\sum_{q=1}^{t} C I I_{i j}^{q} / t$. Plugging Eq. (10) and (20) into it, updated group consensus index can be rewritten as follows: $G C I I_{i j}=1-\sum_{q=1}^{t} \sqrt{\sum_{l=1}^{L}\left(\left(\mu_{i j} \gamma_{i j}^{q l}+\left(1-\mu_{i j}\right) \gamma_{i j}^{c+l}\right)-\sum_{q=1}^{t} w_{i j}^{q}\left(\mu_{i j} \bar{\gamma}_{i j}^{q l}+\left(1-\mu_{i j}\right) \bar{\gamma}_{i j}^{c+l}\right)\right)^{2}} / L t$, 


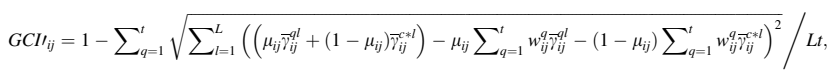

$G C I_{i j}=1-\mu_{i j} \sum_{q=1}^{t} \sqrt{\sum_{l=1}^{L}\left(\bar{\gamma}_{i j}^{q l}-\bar{\gamma}_{i j}^{c * l}\right)^{2}} / L t$.

We have $G C I_{i j}-G C I_{i j}=\left(1-\mu_{i j}\right) \sum_{q=1}^{t} \sqrt{\sum_{l=1}^{L}\left(\bar{\gamma}_{i j}^{q l}-\bar{\gamma}_{i j}^{c t}\right)^{2}} / L t$. Q.E.D.

Since $\mu_{i j} \in[0,1)$, it follows $G C I_{i j}>G C I_{i j}$, which implies that the modification strategy based on Eq. (1) is convergent. In the following, a linear weighting algorithm with optimal feedback weight is developed.

(1) Constraints of feedback mechanism:

With the purpose of consensus improvement, there exist two constraints to control feedback process: satisfying consensus requirement and preserving original attitude. With the former, the proposed feedback mechanism can reach consensus in one iteration. And the latter can retain richness of decision information.

(1.1) Constraint for expected consensus threshold: the updated preference $\bar{h}_{i j}^{q}$ should satisfy consensus requirement. In other words, with updated individual preference $\bar{h}{ }_{i j}^{q}$, the updated group preference $\bar{H} \prime^{c *}=\left(\bar{h}_{i j}^{c *}\right)_{m \times n}$ should be acceptable on all positions:

$$
\begin{aligned}
G C I_{i j}^{*} & =\sum_{j=1}^{n} \sum_{i=1}^{m} C I_{i j}^{*} / m n>E C I_{i j},(i, j) \\
& \in P o s
\end{aligned}
$$

where $C I I_{i j}^{*}$ and $G C I I_{i j}^{*}$ denote the updated individual and group consensus index, respectively. To reduce adjustment range, we prefer the optimal feedback weight $\mu_{i j}^{*}$ making $G C I_{i j}^{*}=E C I_{i j}$ rather than over-adjustment. Let

$f\left(\mu_{i j}\right)=1-\sum_{q=1}^{l} \sqrt{\sum_{l=1}^{L}\left(\mu_{i j} \gamma_{i j}^{q l}+\left(1-\mu_{i j}\right) \gamma_{i j}^{c k}-\gamma_{i j}^{l k}\right)^{2}} / t L-E C I_{i j}$.

Therefore, constraint for expected consensus threshold can be transformed into a zero-point searching process. Zero-point making $f\left(\mu_{i j}\right)=0$ is the optimal feedback weight. According to Theorem 2, it is obvious that $f\left(\mu_{i j}\right)$ is a monotonically decreasing function. Therefore, there is at most one zero point in the feasible region.

Constraint for preserve DMs' attitude. In multiattribute decision process, as mentioned in Remark 1, $0 \leq \gamma^{l}<0.5$ and $0.5<\gamma^{l} \leq 1$ express absolutely opposite attitude. If $\bar{h}_{i j}^{q}$ and $\bar{h}_{i j}^{c *}$ share the same attitude, it is easier for DMs to accept group opinion.

Example 3 Let $\bar{h}_{i j}^{q}=\{0,1,0.2,0.2\}$ and $\bar{h}_{i j}^{c}=\{0,3,0.3$, $0.4\}$ be individual and group preference respectively. Since $0<\bar{\gamma}_{i j}^{q l}, \bar{\gamma}_{i j}^{c l}<0.5$, individual preference has the same attitude with the group. For all $\mu_{i j} \in[0,1]$, DMs would not be distorted by linear weighting process.

However, when individual and group preference show opposite attitude, too small feedback weight can change the original attitude. Therefore, it is necessary to obtain the feasible region of feedback weight.

Example 4 Let $\bar{h}_{i j}^{q}=\{0,2,0.2,0.4\}$ and $\bar{h}_{i j}^{c}=\{0,4,0.6$, $0.7\}$ be individual and group preference, respectively. The updated individual preference is $\bar{h}_{i j}^{q}=\left\{0.2 \mu_{i j}+0.4\left(1-\mu_{i j}\right)\right.$, $\left.0.2 \mu_{i j}+0.6\left(1-\mu_{i j}\right), 0.4 \mu_{i j}+0.7\left(1-\mu_{i j}\right)\right\}$. To remain the original attitude, we have the following constraints: $0.2 \mu_{i j}$ $+0.4\left(1-\mu_{i j}\right) \leq 0.5, \quad 0.2 \mu_{i j}+0.6\left(1-\mu_{i j}\right) \leq 0.5, \quad 0.4 \mu_{i j}+$ $0.7\left(1-\mu_{i j}\right) \leq 0.5$. The feasible region of $\mu_{i j}$ is $[2 / 3,1]$.

During optimization process, we have the following constraint to preserve original attitude:

$$
\begin{aligned}
& \left(\mu_{i j} \bar{\gamma}_{i j}^{q}+\left(1-\mu_{i j}\right) \bar{\gamma}_{i j}^{c *}-0.5\right)\left(\bar{\gamma}_{i j}^{q}-0.5\right) \geq 0, l \\
& \quad=1,2, \ldots, L, q \in T
\end{aligned}
$$

Specifically, the feasible region of $\mu_{i j}$ is $\left[\mu_{i j}^{-}, 1\right]$ and $\mu_{i j}^{-}=\max \left\{\mu_{i j}^{q l} \mid q \in T, l=1,2, \ldots, L\right\}$, where

$$
\mu_{i j}^{q l}=\left\{\begin{array}{l}
\left(0.5-\gamma_{i j}^{c l}\right) /\left(\gamma_{i j}^{q l}-\gamma_{i j}^{c l}\right), \gamma_{i j}^{q l}>0.5>\gamma_{i j}^{c l} \text { or } \gamma_{i j}^{c l}>0.5>\gamma_{i j}^{q l} \\
0, \text { otherwise }
\end{array}\right.
$$

(2) Optimal linear weighting algorithm design

: With the above two constraints, the dichotomybased optimal weight searching algorithm and its flow chart (seen in Fig. 1) are as follows:

Within the above algorithm, Step 3 is to judge 


\section{Algorithm I:}

Input: Individual preference $\bar{H}^{q}=\left(\bar{h}_{i j}^{q}\right)_{m \times n}(q \in T)$, group preference $\bar{H}^{c *}=\left(\bar{h}_{i j}^{c *}\right)_{m \times n}$, adjusted position Pos, limitation of feedback weight $\left[\mu_{i j}^{-}, 1\right],(i, j) \in$ Pos, $q \in T$, maximum error $\varepsilon$.

Output: Optimal feedback weight $\mu_{i j}^{*}$.

For each position to be adjusted $\left(i_{k}, j_{k}\right) \in \operatorname{Pos}, k=1,2, \ldots, K$ :

Step 1: Let $S=\left\{\left(i_{k}, j_{k}, q\right) \mid\left(i_{k}, j_{k}\right) \in \operatorname{Pos}, q \in T\right\}$;

Step 2: Construct updated preference by Eq.(19);

Step 3: Let $\mu_{i_{k} j_{k}}=\mu_{i_{k} j_{k}}^{-}$and use Eq.(10),(17),(20) to calculate $G C I_{i_{k} j_{k}}^{\prime *}$, if $G C I_{i_{k} j_{k}}^{\prime *}>E C I_{i_{k} j_{k}}$, turn to next; otherwise turn to Step 10;

Step 4: Let iteration number $r=1, a_{i_{k} j_{k}}=1$ and $b_{i_{k} j_{k}}=\mu_{i_{k} j_{k}}^{-}$;

Step 5: Set feedback weight of $r$ th iteration: $\mu_{i_{k} j_{k} r}=\left(a_{i_{k} j_{k}}+b_{i_{k} j_{k}}\right) / 2$;

Step 6: Use $\mu_{i_{k} j_{k} r}$ to update preference: $\bar{h}_{i_{k} j_{k} r}^{q}$ and calculate $G C I_{i_{k} j_{k} r}^{*^{*}}$;

Step 7: If $\left(a_{i_{k} j_{k}}-b_{i_{k} j_{k}}\right)<\varepsilon$ for all $\left(i_{k}, j_{k}, q\right) \in S$, output optimal feedback weight $\mu_{i_{k} j_{k} r}$ and turn to Step 11; otherwise, turn to next;

Step 8: If $G C I_{i_{k} j_{k} r}^{\prime *}>E C I_{i_{k} j_{k}}+\delta, b_{i_{k} j_{k}}=\mu_{i_{k} j_{k} r}$; otherwise $a_{i_{k} j_{k}}=$ $\mu_{i_{k} j_{k} r}$

Step 9: Let $r=r+1$ and turn back to Step 5;

Step 10: End searching and let $\mu_{i_{k} j_{k} r}=\mu_{i_{k} j_{k}}^{-},\left(i_{k}, j_{k}, q\right) \in S$;

Step 11: End searching and output optimal feedback weight $\mu_{i_{k} j_{k} r}^{*}$;

whether there exists zero point in feasible region. If there exists, searching process will be activated. Otherwise, minimum feedback weight will be recommended to DMs. In some situations, where urgent consensus is required, the original attitude constraint can be relaxed. Step 7 sets a maximum error $\varepsilon$ to control the searching process. In Step 8, $\delta>0$ is a small positive number to ensure $G C I I_{i_{k} j_{k} r}^{*}>E C I_{i_{k} j_{k}}$.

(3) Time complexity analysis:

In the following, we analyze the time complexity of dichotomy-based optimal linear weighting algorithm. For each position, in best situation, where $\mu_{i_{k} j_{k}}=\mu_{i_{k} j_{k}}^{-}$is the optimal feedback weight, time complexity is $O(1)$. In general situation, searching process will continue until $\left(a_{i_{k} j_{k}}-b_{i_{k} j_{k}}\right)<\varepsilon$.Maximum error constraint is activated through $R$ iterations which satisfy $0.5^{R}\left(1-\mu_{i j}^{-}\right)<\varepsilon$. The minimum iteration number is $R=\log _{2}\left(1-\mu_{i j}^{-}\right)-\log _{2} \varepsilon$.For all conditions, the number of comprehensive iteration is $K \log _{2}\left(1-\mu_{i j}^{-}\right)-K \log _{2} \varepsilon$, where $K$ is the number of positions adjusted. Its iteration number is mainly determined by the feasible range of feedback weight and maximum error. If we relax the original attitude constraint, the iteration number is $\log _{2}\left(1 / \varepsilon^{K}\right)$. The time complexity of dichotomybased linear sum algorithm is $O\left(-K \log _{2} \varepsilon\right)$.

\subsection{Dynamic Hesitant Fuzzy Information Fusion Process}

Based on the above analysis, the proposed two-stage hesitant fuzzy information fusion process and flow chart (seen in Fig. 2) are shown as follows.

The flow chart of whole process is shown as follows: 
Algorithm II:

Step 1: DMs form decision matrixes $H^{q}=\left(h_{i j}^{q}\right)_{m \times n}(q \in T)$;

Step 2: Utilize model $(M-1)$ to obtain normalized hesitant fuzzy matrixes $\bar{H}^{q}=\left(\bar{h}_{i j}^{q}\right)_{m \times n}(q \in T)$;

Step 3: Use model $(M-2)$ to calculate individual consensus range $\left[C I_{i j}^{q-}, C I_{i j}^{q+}\right]$;

Step 4: Estimate consensus threshold $E C I_{i j}$ through statistic inference (Eq.(11-15));

Step 5: Apply model $(M-3)$ to obtain optimal weight $w_{i j}^{q *}$ and group preference $\bar{H}^{c *}=\left(\bar{h}_{i j}^{c *}\right)_{m \times n}$;

Step 6: Calculate group consensus index $G C I_{i j}$, if $G C I_{i j}>E C I_{i j}$ for all $i \in M, j \in N$, output final group opinion $\bar{H}^{c *}=\left(\bar{h}_{i j}^{c *}\right)_{m \times n}$ and turn to Step 10; otherwise, turn to next;

Step 7: Utilize IR to identify positions to be adjusted;

Step 8: Apply Algorithm I to search optimal feedback weight $\mu_{i j}^{*}$;

Step 9: Construct updated group preference $\bar{H}^{\prime c *}=\left(\bar{h}_{i j}^{\prime c *}\right)_{m \times n}$

Step 10: Hesitant fuzzy information fusion complete.

\section{Numerical Example and Comparison Analysis}

In this section, an illustrative example about supplier selection and comparison analysis is illustrated the implementation of the proposed hesitant fuzzy information fusion framework.

\subsection{Background Information}

Since the end of 2019, the large-scale outbreak of COVID19 has shown its destructive impact on many important fields. The pandemic has put tremendous pressure on the public health system. Moreover, it has indirectly influenced the education system, commodity trade, and international logistics due to continued community and border blockade. Compared with the other epidemics, it is more difficult to trace the spreading path of COVID-19 due to the following five features: strong camouflage, long incubation period, various transmission routes, high recurrence rate, and substantial variability. Vaccination is the most effective and efficient method to realize herd immunity. Since there exist differences among vaccine suppliers on the production capacity, vaccine security, etc., it is necessary to evaluate the priority of suppliers to provide the basis for supplier selection and vaccine purchase.

According to the research on the vaccine evaluation and management, the criteria hierarchy of supplier selection is shown as follows: Production capacity $C_{1}$ refers to the ability to satisfy the public demand, specifically containing expected production volume, quality management level, and technical level. Benefit criterion; Vaccine security $C_{2}$ generally contains the protective effect, side effect, vaccine accessibility, etc. Benefit criterion; Supply chain management capacity $C_{3}$ refers to transportation supportability, raw material control ability, and demand response capability, etc. Benefit criterion. In China, there are four main vaccine suppliers: $a_{1}, a_{2}, a_{3}$, and $a_{4}$. The four alternative vaccine suppliers are evaluated based on the above criteria with hesitant fuzzy elements.

In order to obtain the comprehensive evaluation perspectives, four experts majoring in medicine, public health, supply chain management, and public policy are invited to provide preference values. HFE $h_{i j}^{q}$ is applied to represent the $e_{q}$ 's preference on $a_{i}$ under $C_{j}$. Due to the difference in knowledge structure, DMs may have different understandings of the same criterion. In $h_{i j}^{q}$, the values $\gamma_{i j}^{q l}$ represents the preference value of the sub-criterion of $C_{j}$. Due to the diversity of DMs' knowledge structure, the individual understanding to the same criterion leads to the difference of HFEs length, which needs a normalization process for further information fusion. 


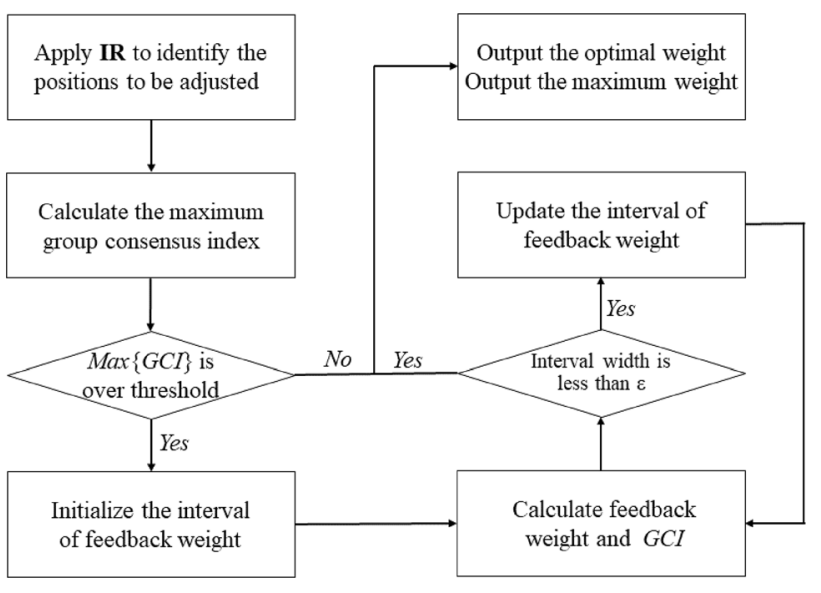

Fig. 1 Flow of optimal feedback weight searching algorithm

\subsection{Numerical Example}

Suppose there are four possible alternatives $A=$ $\left\{a_{1}, a_{2}, a_{3}, a_{4}\right\}$ and three attributes $C=\left\{c_{1}, c_{2}, c_{3}\right\}$, and four experts $E=\left\{e_{1}, e_{2}, e_{3}, e_{4}\right\}$ are invited to make evaluation with HFE. The group consensus is built as following steps:

Step 1: DMs provide evaluation matrix $H^{q}=$ $\left(h_{i j}^{q}\right)_{m \times n}(q=1,2,3,4)$ :

$H_{1}=\left[\begin{array}{ccc}\{0.1,0.2,0.3\} & \{0.3,0.6\} & \{0.4,0.6,0.7\} \\ \{0.2,0.4\} & \{0.5,0.7,0.8\} & \{0.6,0.7,0.9\} \\ \{0.4,0.6\} & \{0.3,0.5,0.6\} & \{0.3,0.3,0.3\} \\ \{0.2,0.3,0.6\} & \{0.2,0.3,0.7\} & \{0.2,0.5,0.6\}\end{array}\right]$

$H_{2}=\left[\begin{array}{ccc}\{0.4,0.6\} & \{0.5,0.6,0.7\} & \{0.3,0.4\} \\ \{0.4,0.5,0.6\} & \{0.1,0.2,0.4\} & \{0.1,0.3,0.6\} \\ \{0.4,0.8\} & \{0.4,0.6\} & \{0.2,0.4,0.7\} \\ \{0.7,0.7,0.7\} & \{0.3,0.5\} & \{0.3,0.6,0.7\}\end{array}\right]$

$H_{3}=\left[\begin{array}{ccc}\{0.3,0.5,0.8\} & \{0.5,0.7,0.8\} & \{0.2,0.3\} \\ \{0.1,0.3,0.5\} & \{0.4,0.6,0.7\} & \{0.2,0.4\} \\ \{0.3,0.6\} & \{0.6,0.7\} & \{0.6,0.7,0.8\} \\ \{0.3,0.4,0.5\} & \{0.1,0.2\} & \{0.1,0.3,0.5\}\end{array}\right]$

$; H_{4}=\left[\begin{array}{ccc}\{0.3,0.4,0.6\} & \{0.2,0.3,0.7\} & \{0.1,0.4\} \\ \{0.4,0.6,0.7\} & \{0.4,0.7\} & \{0.3,0.3,0.3\} \\ \{0.2,0.3,0.5\} & \{0.6,0.6,0.6\} & \{0.3,0.6,0.7\} \\ \{0.2,0.6\} & \{0.2,0.4,0.5\} & \{0.3,0.5\}\end{array}\right]$

Step 2: Utilize model (M-1) to obtain normalized hesitant fuzzy matrixes $\bar{H}^{q}=\left(\bar{h}_{i j}^{q}\right)_{m \times n}(q \in T)$. Apply model (M-1) to maximize proximity index of the group and obtain the normalization parameters. the normalized form of the above HFEs is $h_{12}^{1}=\{0.3,0.516,0.6\}, \quad h_{21}^{1}=$ $\{0.2,0.381,0.4\}, \quad h_{31}^{1}=\{0.4,0.412,0.6\}, \quad h_{11}^{2}=\{0.4,0$.

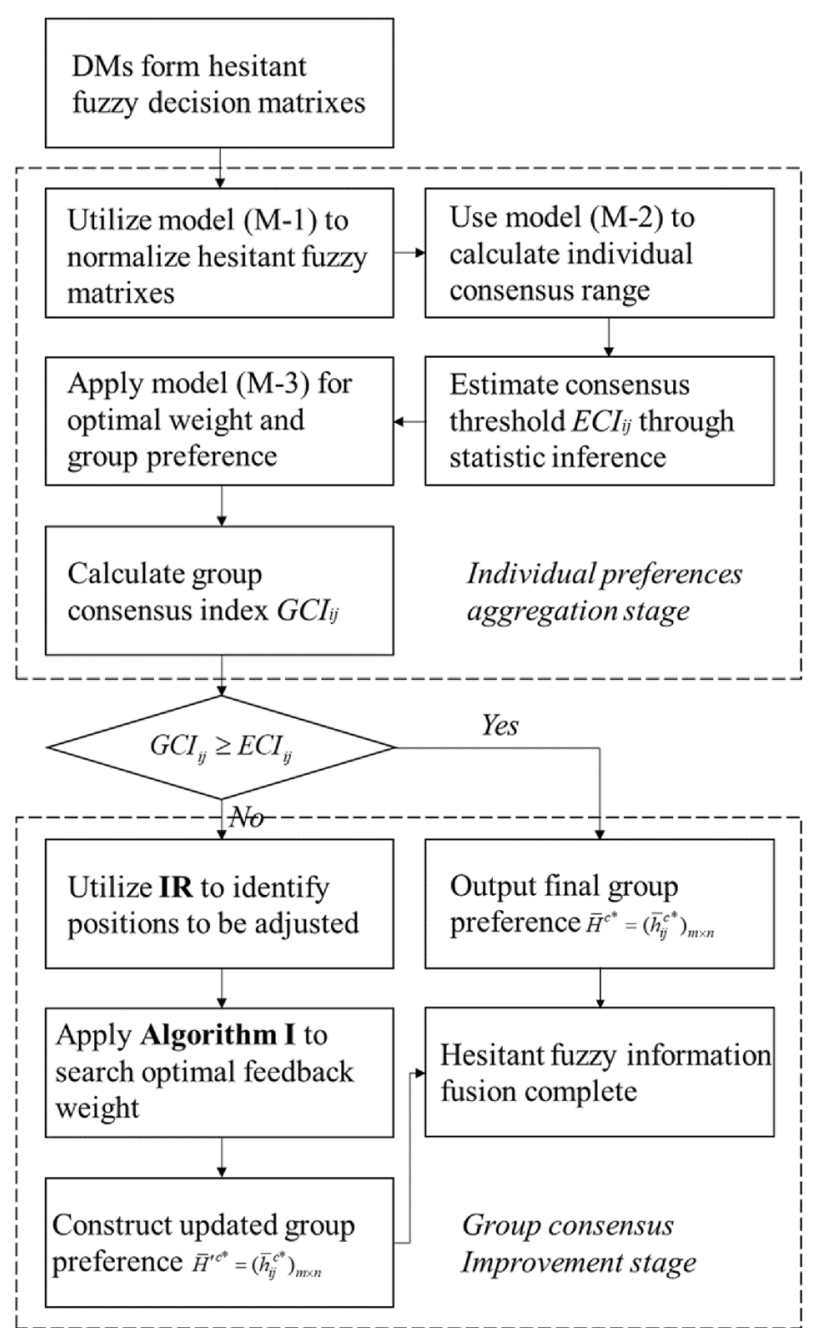

Fig. 2 Flow chart of the proposed information fusion

422, 0.6 $\}, h_{13}^{2}=\{0.3,0.364,0.4\}, h_{31}^{2}=\{0.4,0.417,0.8\}$, $h_{32}^{2}=\{0.4,0.547,0.6\}, \quad h_{42}^{2}=\{0.3,0.347,0.5\}, \quad h_{13}^{3}=$ $\{0.2,0.294,0.3\}, \quad h_{23}^{3}=\{0.2,0.337,0.4\}, \quad h_{31}^{3}=\{0.3$, $0.382, \quad 0.6\}, \quad h_{32}^{3}=\{0.6,0.611,0.7\}, \quad h_{42}^{3}=\{0.1,0.193$, $0.2\}, h_{13}^{4}=\{0.1,0.354,0.4\}, h_{22}^{4}=\{0.4,0.600,0.7\}, h_{41}^{4}$ $=\{0.2,0.339,0.6\}$, and $h_{43}^{4}=\{0.3,0.463,0.5\}$.

The maximum proximity levels $P I_{i j},(i \in M, j \in N)$ are $P I_{11}=0.885, P I_{12}=0.894, P I_{13}=0.892, P I_{21}=0.896$, $P I_{22}=0.874, P I_{23}=0.836, P I_{31}=0.926, P I_{32}=0.927$, $P I_{33}=0.861, P I_{41}=0.880, P I_{42}=0.897$, and $P I_{43}=$ 0.918 .

Step 3: Use model (M-2) to calculate possible individual consensus intervals. The proximity matrix of each DM is 


$$
\begin{aligned}
P I^{1} & =\left[\begin{array}{lll}
0.832 & 0.907 & 0.841 \\
0.900 & 0.877 & 0.752 \\
0.938 & 0.915 & 0.822 \\
0.904 & 0.891 & 0.937
\end{array}\right] \\
P I^{2} & =\left[\begin{array}{lll}
0.909 & 0.910 & 0.916 \\
0.908 & 0.786 & 0.856 \\
0.910 & 0.938 & 0.879 \\
0.798 & 0.918 & 0.908
\end{array}\right] \\
P I^{3} & =\left[\begin{array}{lll}
0.880 & 0.890 & 0.905 \\
0.888 & 0.916 & 0.876 \\
0.945 & 0.925 & 0.849 \\
0.903 & 0.856 & 0.898
\end{array}\right] \\
P I^{4} & =\left[\begin{array}{lll}
0.918 & 0.867 & 0.906 \\
0.885 & 0.916 & 0.856 \\
0.912 & 0.930 & 0.892 \\
0.909 & 0.920 & 0.928
\end{array}\right] .
\end{aligned}
$$

Therefore, based on P-IHFOWA operator and model (M-2) to calculate individual consensus interval $\left[C I_{i j}^{q-}, C I_{i j}^{q+}\right]$ are

$$
\begin{array}{r}
C I^{1}=\left[\begin{array}{lll}
{[0.858,0.862]} & {[0.920,0.951]} & {[0.868,0.889]} \\
{[0.897,0.933]} & {[0.927,0.942]} & {[0.754,0.840]} \\
{[0.965,0.971]} & {[0.931,0.963]} & {[0.833,0.855]} \\
{[0.921,0.986]} & {[0.925,0.935]} & {[0.986,1.000]}
\end{array}\right] \\
C I^{2}=\left[\begin{array}{lll}
{[0.960,0.966]} & {[0.960,1.000]} & {[0.968,1.000]} \\
{[0.958,1.000]} & {[0.805,0.821]} & {[0.915,0.934]} \\
{[0.924,0.941]} & {[0.967,1.000]} & {[0.925,0.937]} \\
{[0.791,0.861]} & {[0.962,0.969]} & {[0.939,0.942]}
\end{array}\right] \\
C I^{3}=\left[\begin{array}{lll}
{[0.925,0.930]} & {[0.931,0.952]} & {[0.928,0.947]} \\
{[0.875,0.915]} & {[0.984,1.000]} & {[0.910,1.000]} \\
{[0.982,1.000]} & {[0.922,0.954]} & {[0.908,0.918]} \\
{[0.948,0.966]} & {[0.874,0.884]} & {[0.918,0.921]}
\end{array}\right] \\
C I^{4}=\left[\begin{array}{lll}
{[0.992,1.000]} & {[0.858,0.894]} & {[0.933,0.943]} \\
{[0.924,0.952]} & {[0.984,1.000]} & {[0.884,0.951]} \\
{[0.930,0.945]} & {[0.931,0.960]} & {[0.971,1.000]} \\
{[0.929,1.000]} & {[0.972,1.000]} & {[0.951,0.963]}
\end{array}\right]
\end{array}
$$

Step 4: Estimate consensus threshold $E C I_{i j}$ through statistic inference. Based on Eq. (11) and (12), we get the average value and variance of each position: $\overline{C I}_{11}=0.937$, $\overline{C I}_{12}=0.933, \overline{C I}_{13}=0.935, \overline{C I}_{21}=0.932, \overline{C I}_{22}=0.933$, $\overline{C I}_{23}=0.898, \overline{C I}_{31}=0.957, \overline{C I}_{32}=0.953, \overline{C I}_{33}=0.918$, $\overline{C I}_{41}=0.925, \quad \overline{C I}_{42}=0.940$, and $\overline{C I}_{43}=0.952 ; s_{11}^{2}=$ $0.0029, \quad s_{12}^{2}=0.0019, \quad s_{13}^{2}=0.0017, \quad s_{21}^{2}=0.0015, \quad s_{22}^{2}$ $=0.0062, \quad s_{23}^{2}=0.0056, \quad s_{31}^{2}=0.0007, \quad s_{32}^{2}=0.0006$, $s_{33}^{2}=0.0030, \quad s_{41}^{2}=0.0048, \quad s_{42}^{2}=0.0020, \quad$ and $\quad s_{43}^{2}=$ 0.0008 .

With Eq. (13-15), the expected consensus thresholds interval $\left[E C I_{i j}^{-}, E C I_{i j}^{+}\right], i \in M, j \in N$ are

$E C I=\left[\begin{array}{lll}{[0.940,0.945]} & {[0.925,0.953]} & {[0.931,0.947]} \\ {[0.910,0.943]} & {[0.939,0.954]} & {[0.892,0.959]} \\ {[0.946,0.958]} & {[0.930,0.961]} & {[0.918,0.929]} \\ {[0.925,0.988]} & {[0.937,0.949]} & {[0.949,0.960]}\end{array}\right]$

Supposing $\eta=0.8$. The expected consensus thresholds are

$E C I_{11}=0.941, E C I_{12}=0.931, E C I_{13}=0.934, E C I_{21}$ $=0.917, E C I_{22}=0.942, E C I_{23}=0.906, E C I_{31}=0.948$, $E C I_{32}=0.937, E C I_{33}=0.920, E C I_{41}=0.938, E C I_{42}=$ 0.940 , and $E C I_{43}=0.952$.

Step 5: Apply model (M-3) to obtain optimal weight $w_{i j}^{q *}$ and group preference $\bar{H}^{c *}=\left(\bar{h}_{i j}^{c *}\right)_{m \times n}$. Without losing generality, $\alpha=\beta=0.88, \lambda=2.25$. The optimal weight matrices of four DMs and group preference $\bar{H}^{c *}=\left(\bar{h}_{i j}^{c *}\right)_{m \times n}$ are

$$
\begin{aligned}
w^{1}= & {\left[\begin{array}{lll}
0.087 & 0.253 & 0.096 \\
0.251 & 0.000 & 0.078 \\
0.000 & 0.107 & 0.129 \\
0.167 & 0.213 & 0.618
\end{array}\right] w^{2} } \\
= & {\left[\begin{array}{lll}
0.166 & 0.267 & 0.600 \\
0.255 & 0.000 & 0.111 \\
0.000 & 0.577 & 0.209 \\
0.084 & 0.247 & 0.118
\end{array}\right] w^{3} } \\
= & {\left[\begin{array}{lll}
0.114 & 0.244 & 0.127 \\
0.248 & 1.000 & 0.651 \\
1.000 & 0.132 & 0.156 \\
0.117 & 0.190 & 0.094
\end{array}\right] w^{4} } \\
= & {\left[\begin{array}{lll}
0.632 & 0.235 & 0.177 \\
0.247 & 0.000 & 0.159 \\
0.000 & 0.183 & 0.505 \\
0.632 & 0.350 & 0.169
\end{array}\right], } \\
H^{c}= & {\left[\begin{array}{lll}
\{0.302,0.401,0.612\} & \{0.392,0.553,0.708\} & \{0.267,0.382,0.428\} \\
\{0.287,0.457,0.564\} & \{0.400,0.600,0.700\} & \{0.248,0.367,0.489\} \\
\{0.300,0.382,0.600\} & \{0.463,0.561,0.615\} & \{0.317,0.552,0.686\} \\
\{0.274,0.382,0.599\} & \{0.208,0.330,0.509\} & \{0.221,0.491,0.590\}
\end{array}\right] . }
\end{aligned}
$$

Step 6: Calculate group consensus index $G C I_{i j}$ with Eq. (17): $G C I_{11}=0.937, G C I_{12}=0.934, G C I_{13}=0.936$, $G C I_{21}=0.932, G C I_{22}=0.937, G C I_{23}=0.907, G C I_{31}=$ $0.958, \quad G C I_{32}=0.953, G C I_{33}=0.919, \quad G C I_{41}=0.930$, $G C I_{42}=0.940$, and $G C I_{43}=0.953$. There exists $G C I_{11}$ $<E C I_{11}=0.941, G C I_{22}<E C I_{22}=0.942, G C I_{33}<E C I_{33}$ $=0.920$, and $G C I_{41}<E C I_{41}=0.938$.

Therefore, turn to Step 7.

Step 7: Apply IR to identify positions to be adjusted: Pos $=\{(1,1),(2,2),(2,3),(4,1)\}$. 


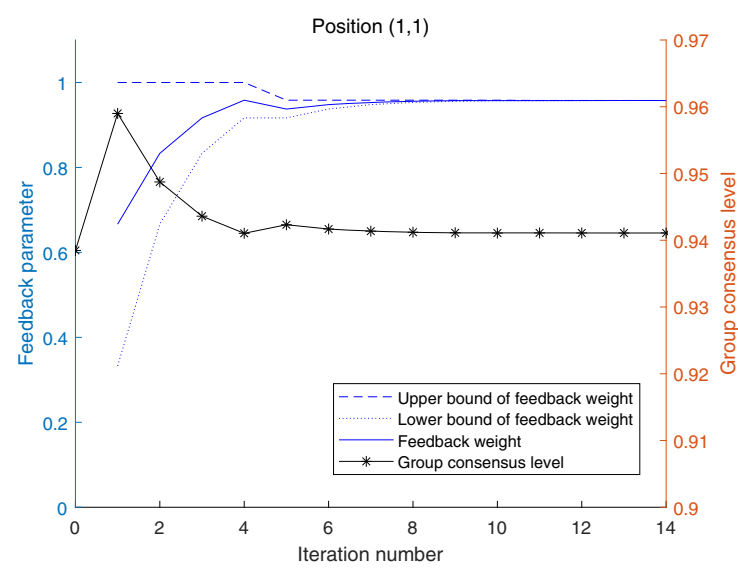

(a) Consensus level and optimal feedback weight in position $(1,1)$

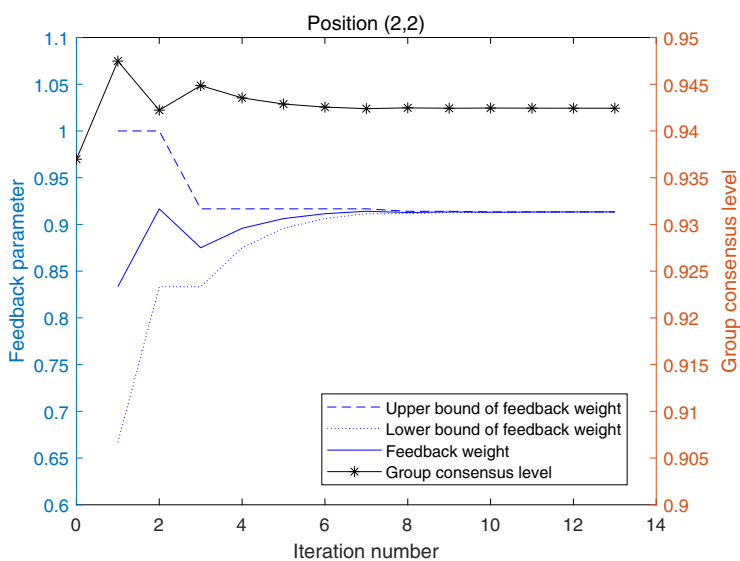

(c) Consensus level and optimal feedback weight in position $(2,2)$

Fig. 3 Iteration process of optimal parameter

Step 8: Apply Algorithm I to search optimal feedback weight $\mu_{i j}^{*}$. First, the limitation of feedback weight is calculated with Eq. (20): $\mu_{11}^{1} \in[0.333,1], \mu_{11}^{2} \in[0,1], \mu_{11}^{3}$ $\in[0,1], \mu_{11}^{4} \in[0,1], \mu_{41}^{1} \in[0,1], \mu_{41}^{2} \in[0.6,1], \mu_{41}^{3} \in[0,1]$, $\mu_{41}^{4} \in[0,1], \mu_{22}^{1} \in[0,1], \mu_{22}^{2} \in[0.666,1], \mu_{22}^{3} \in[0,1], \mu_{22}^{4}$ $\in[0,1], \quad \mu_{33}^{1} \in[0.5,1], \quad \mu_{33}^{2} \in[0.5,1], \quad$ and $\mu_{33}^{3} \in[0,1]$. Therefore, $\mu_{11} \in[0.333,1], \mu_{41} \in[0.600,1], \mu_{22} \in[0.666$, $1]$, and $\mu_{33} \in[0.500,1]$.

Supposing that the maximum error $\varepsilon=0.0001$. In the following, the consensus improvement process of position $(2,2)$ is shown as an example. Other positions can be enhanced similarly.

Step 8.1: Let $S=\{(2,2,1),(2,2,2),(2,2,3),(2,2,4)\}$;

Step 8.2: Construct updated preference:

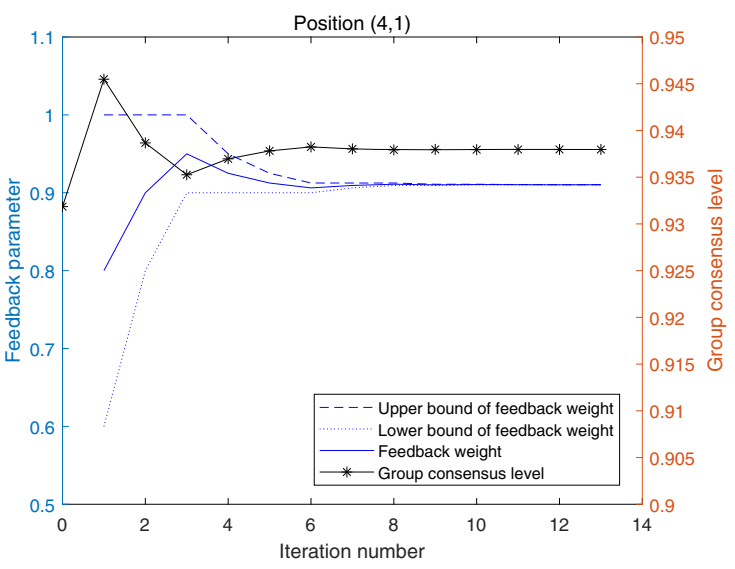

(b) Consensus level and optimal feedback weight in position $(4,1)$

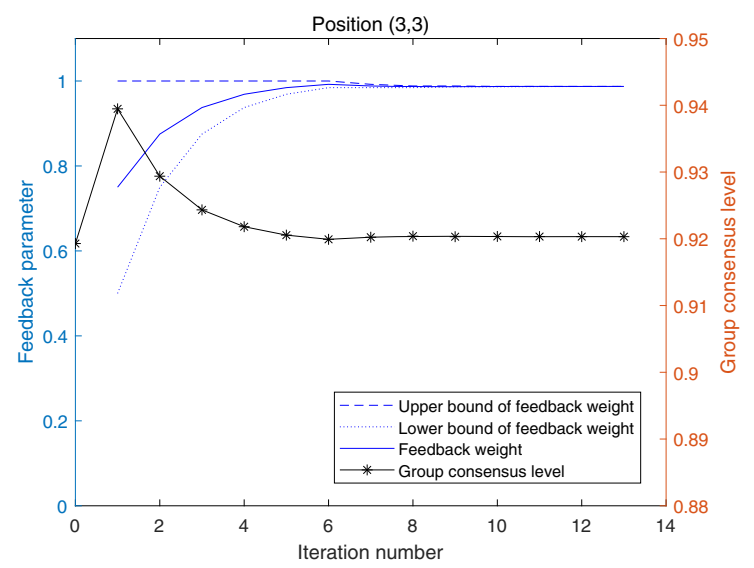

(d) Consensus level and optimal feedback weight in position $(3,3)$

$$
\begin{aligned}
& \bar{h} \prime_{22}^{1}=\mu_{22}\{0.5,0.7,0.8\}+\left(1-\mu_{22}\right)\{0.4,0.6,0.7\}, \bar{h} \prime_{22}^{2} \\
&= \mu_{22}\{0.1,0.2,0.4\}+\left(1-\mu_{22}\right)\{0.4,0.6,0.7\} . \\
& \bar{h} \prime_{22}^{3}=\mu_{22}\{0.4,0.6,0.7\}+\left(1-\mu_{22}\right)\{0.4,0.6,0.7\}, \text { and } \\
& \bar{h} \prime_{22}^{4}=\mu_{22}\{0.4,0.6,0.7\}+\left(1-\mu_{22}\right)\{0.4,0.6,0.7\} .
\end{aligned}
$$

Step 8.3: Let $\mu_{22}=0.666$ and use Eq. (10), (21), and (22) to calculate group consensus index. Since $G C I_{22}^{*}=$ $0.958>E C I_{22}$ turn to Step 8.4;

Step 8.4: Let iteration number $r=1, a_{22}=1$, and $b_{22}=0.666$;

Step 8.5: Set feedback weight of the 1st iteration: $\mu_{22 r}=\left(a_{22}+b_{22}\right) / 2=0.833$;

Step 8.6: Use $\mu_{22 r}=0.333$ to update preference: $\bar{h} \prime_{22}^{1}=\{0.433,0.633,0.733\}, \bar{h} \prime_{22}^{2}=\{0.300,0.666,0.600\}$, $\bar{h}_{22}^{3}=\bar{h}_{22}^{4}=\{0.4,0.6,0.7\}$. Apply model (M-3) and Eq. (17) to calculate $G C I_{22 r}^{*}=0.9451>E_{22}$; 
Step 8.9: Since $G C I_{22 r}^{*}=0.9451>E C I_{22}$ and $b_{22}-$ $a_{22}>\varepsilon$, let $b_{22}=0.833$;

Step 8.8: Let $r=r+1$ and turn back to Step 8.5;

After 13 iterations, $b_{22}-a_{22}=0.00008<\varepsilon$. The optimal feedback weight is $\mu_{22}^{*}=0.8836, G C I I_{22 r}^{*}=0.9426$ $>E C I_{22}$. Similar as above process, the optimal feedback weights are $\mu_{11}^{*}=0.9575, \mu_{41}^{*}=0.9103, \mu_{22}^{*}=0.9133$, and $\mu_{33}^{1 *}=0.9872$. The improved group consensus indexes are $G C I_{11}^{\prime}=0.9412>E C I_{11}, G C I_{41}^{\prime}=0.9381>E C I_{41}$, $G C I_{22}^{\prime}=0.9426>E C I_{22}$, and $G C I_{33}^{\prime}=0.9205>E C I_{33}$. There are four positions involved into feedback process. The iteration process is shown in Fig. 3:

Step 9: Use optimal feedback weight to construct updated group preference $\bar{H} \prime^{c *}=\left(\bar{h} c_{i j}^{c *}\right)_{m \times n}$ :

$H \prime^{c}=\left[\begin{array}{lll}\{0.302,0.400,0.610\} & \{0.391,0.553,0.707\} & \{0.266,0.382,0.427\} \\ \{0.286,0.457,0.564\} & \{0.400,0.600,0.700\} & \{0.250,0.368,0.490\} \\ \{0.300,0.382,0.600\} & \{0.463,0.561,0.615\} & \{0.312,0.560,0.689\} \\ \{0.282,0.387,0.596\} & \{0.208,0.330,0.509\} & \{0.221,0.491,0.589\}\end{array}\right]$

Step 10: Consensus completed.

\subsection{Discussion and Comparison Analysis}

First, the rationality of expected consensus threshold estimation process is explained through simulation method and Kolmogorov-Smirnov test. And then, the proposed method with existing baseline approaches is compared to verify the effectiveness and efficiency of our method. The comparison analysis mainly focuses on preference aggregation effectiveness and adjustment distance.

(1) Consensus sequence distribution discussion:

First of all, stochastic simulation is applied to illustrate rationality of the assumption that individual consensus sequence $\left\{C I_{i j}^{1-}, C I_{i j}^{1+}, C I_{i j}^{2-}, C I_{i j}^{2+}, \ldots, C I_{i j}^{t-}, C I_{i j}^{t+}\right\}$ obeys normal distribution. Ten groups of hesitant fuzzy decision matrices with $10,20 \ldots, 100 \mathrm{DMs}$, respectively, are produced randomly by Matlab to simulate the group decision-making process. Since the number of DMs involved in GDM usually less than 100, Kolmogorov-Smirnov test (KS test) is applied to verify that individual consensus sequence obeys normal distribution. To ensure reliability of the simulation, all experiments were repeated 100 times.

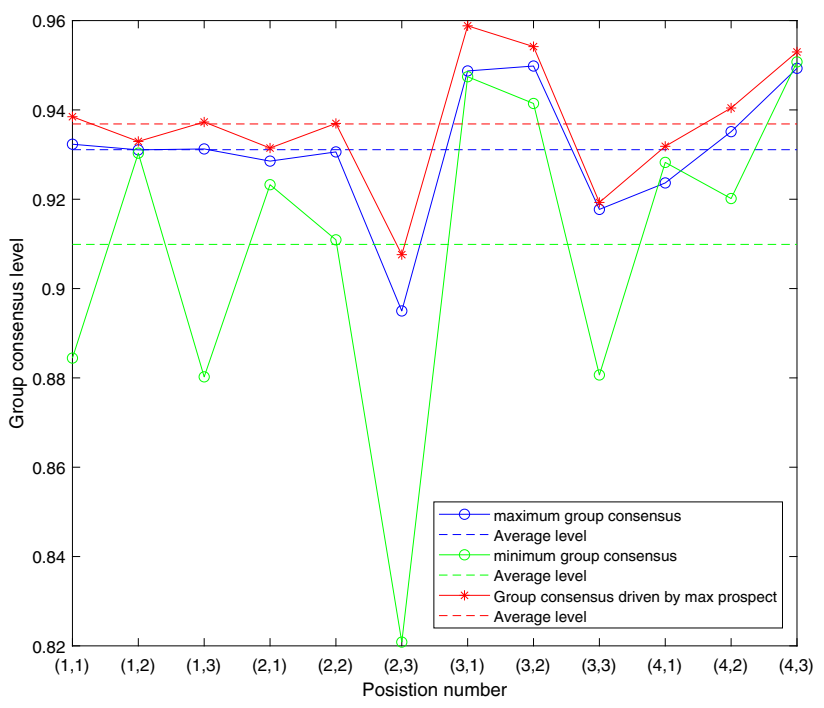

Fig. 4 Group consensus levels of subjective weight

Supposing that significance level is 0.05 . The average confidence levels with different group scale are shown in Table 1.

$H_{0}$ Individual consensus sequence obeys normal distribution;

$H_{1} \quad$ Individual consensus sequence does not obey normal distribution;

When group scale varies from 10 to 100 , the average confidence level is larger than significance level. Therefore, we accept the original hypothesis: Individual consensus sequence obeys normal distribution. Thus, it is reliable to use T-distribution to estimate expected consensus threshold.

(2) The aggregation effectiveness comparison

: From the perspective of individual information aggregation, the weight of DMs is one of the key factors in aggregation. Xu [7] and Zhou [8] thought that the weight of DM is determined by decision moderator. As an exogenous parameter, DM weight would not be influenced by their assessment and compactness of the group. Considering the distance between individual and group, Liu and $\mathrm{Xu}$ [13]

Table 1 Confidence level with different group scales

\begin{tabular}{llllll}
\hline Group scale & 10 & 20 & 30 & 40 & 50 \\
\hline Confidence & 0.1031 & 0.1349 & 0.2241 & 0.2588 & 0.28570 \\
Group scale & 60 & 70 & 80 & 90 & 100 \\
Confidence & 0.5135 & 0.6011 & 0.7212 & 0.8282 & 0.9097 \\
\hline
\end{tabular}




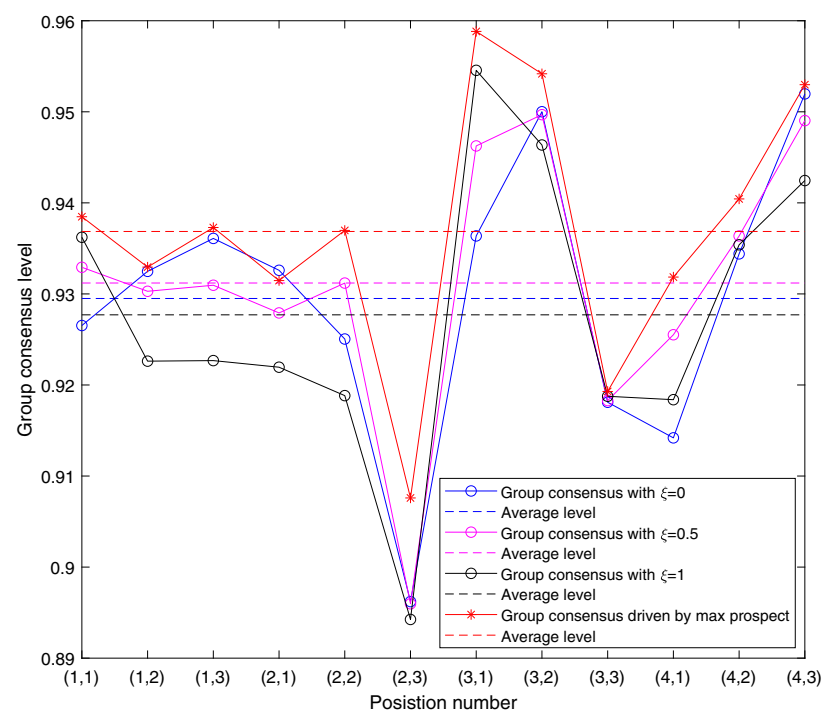

Fig. 5 Group consensus levels of optimization-based weight

proposed an optimization-based weight determination method. Group consensus index $G C I_{i j}$ of each position is compared with subjective weight [7][7] and optimization-based weight [13].

(2.1) To compare with subjective method, 1000 weight vectors are generated randomly to simulate subjective weight and the normalization parameter is $\xi=0.5$. The comparison results regarding the group consensus index that are used in Sect. 5.2 are offered in Fig. 4.

(2.2) According to optimization-based method [13], the optimal weight vectors are calculated under different normalization parameters, which minimize distance between individual and group:

$$
\begin{aligned}
w_{1} & =0.1611, w_{2}=0.1930, w_{3}=0.2644, w_{4} \\
& =0.3759, \xi=0, \\
w_{1} & =0.1541, w_{2}=0.1388, w_{3}=0.2303, w_{4} \\
& =0.4726, \xi=0.5, \\
w_{1} & =0.1321, w_{2}=0.1594, w_{3}=0.2029, w_{4} \\
& =0.5056, \xi=1 .
\end{aligned}
$$

The comparison results are offered in Fig. 5.

From the above two figures, the proposed aggregation method performs better than the two existing methods almost in each position. Due to better acceptability of group preference, less adjustment range will be produced and more original information will be preserved.

(3) Adjustment volume comparison

From consensus improvement, the proposed dichotomybased automatic feedback method is compared with the traditional linear sum method and minimum adjustment method. [7] Global and local feedback methods is used to improve consensus, respectively. Traditional feedback mechanism has not provided adjustment recommendation, which may lead to too much loss of information. To control adjustment distance, [18] and [40] constructed programming model to enhance consensus. However, the original attitude of DMs may be changed during modification. To unify the comparison standard, supposing consensus threshold 0.95 and all DMs are the same important.

(3.1) With traditional feedback method, the feedback weight is usually set by DMs. Local feedback method [10] is applied to improve consensus. Supposing $\mu=0.6$. The updated individual preferences are

$\begin{aligned} H \prime_{1}= & {\left[\begin{array}{lll}\{0.173,0.275,0.425\} & \{0.335,0.529,0.643\} & \{0.343,0.526,0.610\} \\ \{0.234,0.411,0.465\} & \{0.446,0.642,0.751\} & \{0.424,0.530,0.723\} \\ \{0.400,0.411,0.600\} & \{0.300,0.500,0.600\} & \{0.313,0.390,0.446\} \\ \{0.268,0.357,0.602\} & \{0.202,0.306,0.628\} & \{0.200,0.500,0.600\}\end{array}\right] } \\ H / 2 & =\left[\begin{array}{lll}\{0.353,0.409,0.605\} & \{0.455,0.580,0.703\} & \{0.283,0.385,0.430\} \\ \{0.354,0.483,0.585\} & \{0.206,0.342,0.511\} & \{0.244,0.386,0.615\} \\ \{0.400,0.416,0.800\} & \{0.400,0.547,0.600\} & \{0.253,0.450,0.686\} \\ \{0.577,0.605,0.662\} & \{0.261,0.333,0.502\} & \{0.300,0.600,0.700\}\end{array}\right] \\ H \prime_{3}= & {\left[\begin{array}{lll}\{0.293,0.455,0.725\} & \{0.455,0.640,0.763\} & \{0.223,0.343,0.370\} \\ \{0.174,0.363,0.525\} & \{0.386,0.582,0.691\} & \{0.280,0.400,0.543\} \\ \{0.300,0.382,0.600\} & \{0.600,0.610,0.700\} & \{0.433,0.630,0.746\} \\ \{0.337,0.425,0.542\} & \{0.141,0.241,0.322\} & \{0.100,0.300,0.500\}\end{array}\right] } \\ H \prime_{4}= & {\left[\begin{array}{lll}\{0.293,0.395,0.605\} & \{0.275,0.400,0.703\} & \{0.163,0.379,0.430\} \\ \{0.354,0.543,0.645\} & \{0.386,0.582,0.691\} & \{0.316,0.386,0.507\} \\ \{0.200,0.300,0.500\} & \{0.600,0.600,0.600\} & \{0.313,0.570,0.686\} \\ \{0.277,0.388,0.602\} & \{0.201,0.365,0.502\} & \{0.300,0.463,0.500\}\end{array}\right] }\end{aligned}$

(3.2) With minimum adjustment method [18], elastic variables are introduced for programming model with minimum adjustment objective.

$$
\begin{gathered}
H_{1}^{\prime \prime}=\left[\begin{array}{lll}
\{0.415,0.515,0.615\} & \{0.300,0.515,0.600\} & \{0.178,0.378,0.478\} \\
\{0.450,0.631,0.650\} & \{0.171,0.371,0.471\} & \{0.378,0.478,0.678\} \\
\{0.400,0.411,0.600\} & \{0.300,0.500,0.600\} & \{0.566,0.566,0.566\} \\
\{0.511,0.611,0.911\} & \{0.029,0.129,0.529\} & \{0.200,0.500,0.600\}
\end{array}\right], \\
H_{2}^{\prime \prime}=\left[\begin{array}{lll}
\{0.400,0.421,0.600\} & \{0.500,0.600,0.700\} & \{0.300,0.364,0.400\} \\
\{0.400,0.500,0.600\} & \{0.456,0.556,0.756\} & \{0.388,0.588,0.888\} \\
\{0.400,0.416,0.800\} & \{0.400,0.547,0.600\} & \{0.366,0.566,0.866\} \\
\{0.510,0.510,0.510\} & \{0.300,0.346,0.500\} & \{0.300,0.600,0.700\}
\end{array}\right], \\
H_{3}^{\prime \prime}=\left[\begin{array}{lll}
\{0.031,0.231,0.531\} & \{0.309,0.509,0.609\} & \{0.426,0.520,0.526\} \\
\{0.362,0.562,0.762\} & \{0.400,0.600,0.700\} & \{0.504,0.641,0.704\} \\
\{0.300,0.382,0.600\} & \{0.600,0.610,0.700\} & \{0.332,0.532,0.632\} \\
\{0.583,0.683,0.783\} & \{0.356,0.449,0.456\} & \{0.100,0.300,0.500\}
\end{array}\right], \\
H_{4}^{\prime \prime}=\left[\begin{array}{lll}
\{0.300,0.400,0.600\} & \{0.386,0.486,0.886\} & \{0.322,0.576,0.622\} \\
\{0.187,0.387,0.487\} & \{0.400,0.600,0.700\} & \{0.623,0.623,0.623\} \\
\{0.200,0.300,0.500\} & \{0.600,0.600,0.600\} & \{0.300,0.600,0.700\} \\
\{0.498,0.638,0.898\} & \{0.200,0.400,0.500\} & \{0.300,0.463,0.500\}
\end{array}\right],
\end{gathered}
$$

(3.3) With the proposed dichotomy-based optimal linear sum method, supposing maximum error $\varepsilon=0.0001$. To focus on the adjustment process, we assume that all DMs are the same important. 


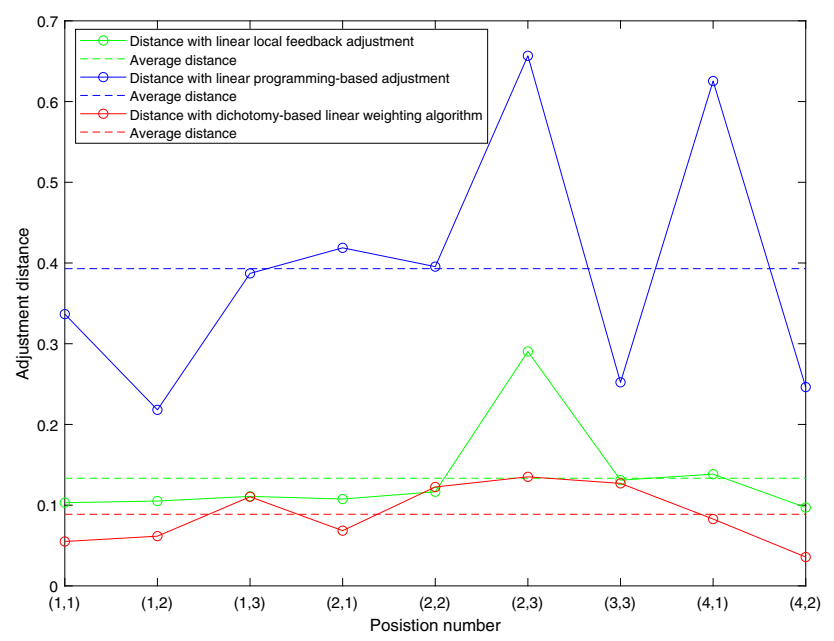

Fig. 6 Adjustment distance of three consensus improvement methods

$$
\begin{gathered}
H_{1}^{\prime \prime \prime}=\left[\begin{array}{lll}
\{0.137,0.237,0.355\} & \{0.369,0.545,0.634\} & \{0.380,0.554,0.641\} \\
\{0.241,0.405,0.441\} & \{0.500,0.700,0.800\} & \{0.600,0.700,0.900\} \\
\{0.400,0.411,0.600\} & \{0.300,0.500,0.600\} & \{0.300,0.356,0.375\} \\
\{0.200,0.300,0.600\} & \{0.200,0.342,0.614\} & \{0.200,0.500,0.600\}
\end{array}\right], \\
H_{2}^{\prime \prime \prime}=\left[\begin{array}{lll}
\{0.400,0.421,0.600\} & \{0.500,0.600,0.700\} & \{0.300,0.364,0.400\} \\
\{0.400,0.500,0.600\} & \{0.201,0.335,0.501\} & \{0.100,0.300,0.600\} \\
\{0.400,0.416,0.800\} & \{0.400,0.547,0.600\} & \{0.200,0.400,0.700\} \\
\{0.582,0.614,0.676\} & \{0.300,0.346,0.500\} & \{0.300,0.600,0.700\}
\end{array}\right], \\
H_{3}^{\prime \prime \prime}=\left[\begin{array}{lll}
\{0.300,0.472,0.744\} & \{0.500,0.700,0.800\} & \{0.200,0.294,0.300\} \\
\{0.201,0.367,0.533\} & \{0.400,0.600,0.700\} & \{0.200,0.337,0.400\} \\
\{0.300,0.382,0.600\} & \{0.600,0.610,0.700\} & \{0.500,0.700,0.800\} \\
\{0.300,0.400,0.500\} & \{0.114,0.222,0.242\} & \{0.100,0.300,0.500\}
\end{array}\right] . \\
H_{4}^{\prime \prime \prime}=\left[\begin{array}{lll}
\{0.300,0.400,0.600\} & \{0.329,0.429,0.700\} & \{0.100,0.354,0.400\} \\
\{0.400,0.600,0.700\} & \{0.400,0.600,0.700\} & \{0.300,0.300,0.300\} \\
\{0.200,0.300,0.500\} & \{0.600,0.600,0.600\} & \{0.300,0.600,0.700\} \\
\{0.200,0.339,0.600\} & \{0.200,0.400,0.500\} & \{0.300,0.463,0.500\}
\end{array}\right] .
\end{gathered}
$$

The comparison results over-adjustment distance between updated preference and the original are offered in Fig. 6. From the above results, the proposed dichotomybased linear sum algorithm performs better than the existing two methods on adjustment distance. The average adjustment distances are dis dichotomy $=0.0887$, dis linear $=$ 0.1059 , and dis programming $=0.3930$, respectively. Considering the original attitude preservation, the proposed method preserves DMs' original attitude. However, the traditional linear sum method and programming-based method can distort original attitude, which can be seen in the above updated preference.

\section{Conclusions}

This paper proposes a novel hesitant fuzzy information fusion method based on prospect theory and dichotomy algorithm. The main contributions of this paper are summarized as follows.
(1) A novel normalization method of HFE is proposed to maximize the compactness of the group, which could avoid the randomness and uncertainty of the traditional normalization method and provide a sound foundation for further information aggregation and consensus improvement.

(2) The individual consensus range is extracted with the P-IOWA operator for expected group consensus threshold estimation based on statistic inference. The objective consensus threshold provides a stable benchmark to control the consensus improvement process.

(3) During the individual preference aggregation process, the prospect function is constructed to depict the potential gain or loss. And the optimal weight vector is assigned to DMs by maximizing comprehensive prospects. Compared with the existing subjective and optimization-based method, the maximum prospect-based weight assignment method can maximize the efficiency of the aggregation process.

(4) Individual acceptance limitation is calculated for consensus improvement. A dichotomy-based linear sum algorithm is designed to search the optimal feedback weight. With the novel consensus improvement method, group consensus requirements can be satisfied without repeated interactions, and individual original attitudes can also be preserved as far as possible.

Meanwhile, we point out some future research directions: In recent years, mobile Internet technology facilitates the large-scale decision-making problem, which has received extensive attention (seen in [29, 34]). Thus, it will be very interesting to extend the research of this paper to large-scale decision-making problems. Moreover, decision information in existing researches is often structured, such as crisp numbers or fuzzy numbers. In a practical situation, the DMs can express their preference with unstructured information, such as text or picture (seen in [41]). It could be very interesting and challenging to combine traditional decisions with big-data analysis technology.

Further research can be improved in the following aspects: (1) Simplify the form of information fusion framework. Specifically, the optimization model in consensus threshold estimation can be revised with a more straightforward form in future research; (2) Identify the position to be adjusted more flexibly. A more flexible IR is expected to identify the positions to be modified to a specific DM, preserving the individual proximity order.

Acknowledgements This work was supported by the Grant (Nos.71971117) from NSF of China and the Grant (Nos.17YJA630035) from the Chinese Ministry of Education. 


\section{References}

1. Antonio, S., Humberto, B.: A wrapper methodology to learn interval-valued fuzzy rule-based classification systems. Appl. Soft Comput. 104, 107249 (2021)

2. Rong, Y., Liu, Y., Pei, Z.: Interval-valued intuitionistic fuzzy generalized bonferroni mean operators for multi-attribute decision making. Int. J. Fuzzy Syst. 4, 1-27 (2021)

3. Zhao, M., Wei, G., Wei, C., Wu, J.: Pythagorean fuzzy todim method based on the cumulative prospect theory for magdm and its application on risk assessment of science and technology projects. Int. J. Fuzzy Syst. 2, 1-15 (2021)

4. Liao, N., Wei, G., Chen, X.: Todim method based on cumulative prospect theory for multiple attributes group decision making under probabilistic hesitant fuzzy setting. Int. J. Fuzzy Syst. 7, 1-18 (2021)

5. Zhang, Z.M., Chen, S.: Group decision making based on acceptable multiplicative consistency and consensus of hesitant fuzzy linguistic preference relations. Inf. Sci. 541, 531-550 (2020)

6. Zhong, X.Y., Xu, X.H.: Clustering-based method for large group decision making with hesitant fuzzy linguistic information: Integrating correlation and consensus. Appl. Soft Comput. 87, $105973(2020)$

7. Xu, Y.J., Wen, X.W., Sun, H., Wang, H.M.: Consistency and consensus models with local adjustment strategy for hesitant fuzzy linguistic preference relations. Int. J. Fuzzy Syst. 20, 2216-2233 (2018)

8. Zhou, X.Y., Wang, L.Q., Liao, H.C., Wang, S.Y., Fujita, H.: A prospect theory-based group decision approach considering consensus for portfolio selection with hesitant fuzzy information. Knowl.-Based Syst. 168, 28-38 (2018)

9. Liu, X., Xu, Y.J., Montes, R., Dong, Y.C., Herrera, F.: Analysis of self-confidence indices-based additive consistency for fuzzy preference relations with self-confidence and its application in group decision making. Int. J. Intell. Syst. 34, 920-946 (2019)

10. Xu, Y.J., Cabrerizo, F., Herrera-Viedma, E.: A consensus model for hesitant fuzzy preference relations and its application in water allocation management. Appl. Soft Comput. 58, 265-284 (2017)

11. Yager, R.R.: Quantifier guided aggregation using owa operators. Int. J. Intell. Syst. 11, 49-73 (1996)

12. Zhang, B.W., Liang, H.M., Zhang, G.Q.: Reaching a consensus with minimum adjustment in magdm with hesitant fuzzy linguistic term sets. Information Fusion 42, 12-23 (2017)

13. Liu, N.N., He, Y., Xu, Z.S.: A new approach to deal with consistency and consensus issues for hesitant fuzzy linguistic preference relations. Appl. Soft Comput. 76, 400-415 (2019)

14. Xiao, J., Wang, X., Zhang, H.J.: Managing classification-based consensus in social network group decision making: An optimization-based approach with minimum information loss. Information Fusion 63, 74-87 (2020)

15. Zhang, Z.M., Pedrycz, W.: A consistency and consensus-based goal programming method for group decision-making with interval-valued intuitionistic multiplicative preference relations. IEEE Transactions on Cybernetics 49, 3640-3654 (2019)

16. Mohammadi, M., Rezaei, J.: Ensemble ranking: Aggregation of rankings produced by different multi-criteria decision-making methods. Omega 96, 102254 (2020)

17. Mohammadi, M., Rezaei, J.: Bayesian best-worst method: A probabilistic group decision making model. Omega 96, 102075 (2020)

18. Wan, S.P., Zhong, L., Dong, J.Y.: A new method for group decision making with hesitant fuzzy preference relations based on multiplicative consistency. IEEE Trans. Fuzzy Syst. 99, 1-12 (2019)
19. Wu, P., Liu, J.P., Zhou, L.G., Chen, H.Y.: Algorithm for improving additive consistency of linguistic preference relations with an integer optimization model. Appl. Soft Comput. 86, 105955 (2020)

20. Cao, M.S., Wu, J., Chiclana, F., Urena, R., Herrera-Viedma, E.: A personalized consensus feedback mechanism based on maximum harmony degree. IEEE Transactions on Systems, Man, and Cybernetics: Systems 243, 713-721 (2019)

21. Pérez, I., Cabrerizo, F., Alonso, S., Dong, Y., Chiclana, F., Herrera-Viedma, E.: On dynamic consensus processes in group decision making problems. Inf. Sci. 459, 20-35 (2018)

22. Tang, M., Zhou, X.Y., Liao, H.C., Xu, J.P., Fujita, H., Herrera, F.: Ordinal consensus measure with objective threshold for heterogeneous large-scale group decision making. Knowl.-Based Syst. 180, 62-74 (2019)

23. Ben-Arieh, D., Easton, T.: Multi-criteria group consensus under linear cost opinion elasticity. Decis. Support Syst. 43, 713-721 (2007)

24. Zhang, H.J., Dong, Y.C., Chiclana, F., Yu, S.: Consensus efficiency in group decision making: A comprehensive comparative study and its optimal design. Eur. J. Oper. Res. 275, 580-598 (2018)

25. Wu, Z.B., Huang, S., Xu, J.P.: Multi-stage optimization models for individual consistency and group consensus with preference relations. Eur. J. Oper. Res. 275, 182-194 (2018)

26. Xiao, J., Wang, X., Zhang, H.J.: Managing personalized individual semantics and consensus in linguistic distribution largescale group decision making. Information Fusion 53, 20-34 (2019)

27. Zhang, B.W., Dong, Y.C., Zhang, H.J., Pedrycz, W.: Consensus mechanism with maximum-return modifications and minimumcost feedback: A perspective of game theory. Eur. J. Oper. Res. 287, 546-559 (2020)

28. Liu, X., Xu, Y.J., Montes, R., Ding, R.X., Herrera, F.: Alternative ranking-based clustering and reliability index-based consensus reaching process for hesitant fuzzy large scale group decision making. IEEE Trans. Fuzzy Syst. 27, 159-171 (2019)

29. Du, Z.J., Yu, S.M., Xu, X.H.: Managing noncooperative behaviors in large-scale group decision-making: Integration of independent and supervised consensus-reaching models. Inf. Sci. 531, $119-138(2020)$

30. Gou, X.J., Xu, Z.S., Liao, H.C., Herrera, F.: Consensus model handling minority opinions and noncooperative behaviors in large-scale group decision-making under double hierarchy linguistic preference relations. IEEE Transactions on Cybernetics 99, $1-14(2020)$

31. Torra, V.: Hesitant fuzzy sets. Int. J. Intell. Syst. 25, 529-539 (2010)

32. Xia, M.M., Xu, Z.S.: Hesitant fuzzy information aggregation in decision making. Int. J. Approximate Reasoning 52, 395-407 (2011)

33. Zhang, X.L., Xu, Z.S., Wang, H.: Heterogeneous multiple criteria group decision making with incomplete weight information: A deviation modeling approach. Information Fusion 25, 49-62 (2015)

34. Chao, X.R., Kou, G., Peng, Y., Viedma, E.H.: Large-scale group decision-making with non-cooperative behaviors and heterogeneous preferences: An application in financial inclusion. Eur. J. Oper. Res. 288, 271-293 (2020)

35. Zhu, B., Xu, Z., Xu, J.: Deriving a ranking from hesitant fuzzy preference relations under group decision making. IEEE Transactions on Cybernetics 44, 1328-1337 (2013)

36. Xu, Z.S., Xia, M.M.: Distance and similarity measures for hesitant fuzzy sets. Inf. Sci. 181, 2128-2138 (2011)

37. Tversky, K.: Prospect theory: An analysis of decision under risk. Econometrica 47, 263-291 (1979) 
38. Wu, A.P., Li, H., Dong, M.: A novel two-stage method for matching the technology suppliers and demanders based on prospect theory and evidence theory under intuitionistic fuzzy environment. Appl. Soft Comput. 95, 106553 (2020)

39. Wang, Z.L., Wang, Y.M.: Prospect theory-based group decisionmaking with stochastic uncertainty and 2-tuple aspirations under linguistic assessments. Information Fusion 56, 81-92 (2020)

40. Li, J., Wang, J.Q., Hu, J.H.: Consensus building for hesitant fuzzy preference relations with multiplicative consistency. Comput. Ind. Eng. 128, 387-400 (2018)

41. Kazmaier, J., Vuuren, J.: A generic framework for sentiment analysis: Leveraging opinion-bearing data to inform decision making. Decis. Support Syst. 135, 113304 (2020)

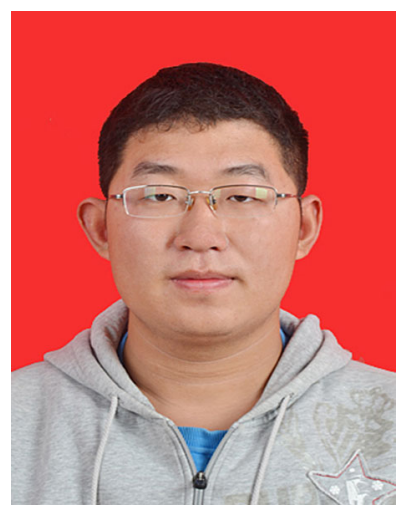

Xiwen Tao received his B.S. degree in Industrial Engineering from Zhengzhou University of Aeronautics, Zhengzhou, China, in 2017. He is currently working toward his Ph.D. degree in Management Science and Engineering with the School of Economics and Management, Nanjing University of Science and Technology, Nanjing, China. His research interests include decision analysis, group decision making, and multi-attribute decision making.

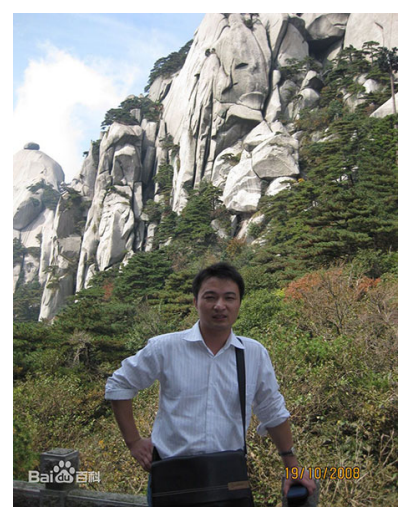

Wenqi Jiang received his M.S. degree and his Ph.D. degree in Management Science and Engineering with the School of Management, University of Science and Technology of China, Hefei, China. His research interests include decision analysis, group decision making, and multi-attribute decision making. 